\title{
Architecture and noise analysis of continuous-variable quantum gates using two- dimensional cluster states
}

\author{
Larsen, Mikkel V.; Neergaard-Nielsen, Jonas S.; Andersen, Ulrik L.
}

Published in:

Physical Review A

Link to article, DOI:

10.1103/PhysRevA.102.042608

Publication date:

2020

Document Version

Publisher's PDF, also known as Version of record

Link back to DTU Orbit

Citation $(A P A)$ :

Larsen, M. V., Neergaard-Nielsen, J. S., \& Andersen, U. L. (2020). Architecture and noise analysis of

continuous-variable quantum gates using two-dimensional cluster states. Physical Review A, 102(4), [042608 ]. https://doi.org/10.1103/PhysRevA.102.042608

\section{General rights}

Copyright and moral rights for the publications made accessible in the public portal are retained by the authors and/or other copyright owners and it is a condition of accessing publications that users recognise and abide by the legal requirements associated with these rights.

- Users may download and print one copy of any publication from the public portal for the purpose of private study or research.

- You may not further distribute the material or use it for any profit-making activity or commercial gain

- You may freely distribute the URL identifying the publication in the public portal 


\title{
Architecture and noise analysis of continuous-variable quantum gates using two-dimensional cluster states
}

\author{
Mikkel V. Larsen $\oplus^{*},{ }^{*}$ Jonas S. Neergaard-Nielsen, and Ulrik L. Andersen ${ }^{\dagger}$ \\ Center for Macroscopic Quantum States (bigQ), Department of Physics, Technical University of Denmark, \\ Fysikvej, 2800 Kongens Lyngby, Denmark
}

(Received 10 June 2020; accepted 28 September 2020; published 19 October 2020)

\begin{abstract}
Due to its unique scalability potential, continuous-variable quantum optics is a promising platform for large-scale quantum computing. In particular, very large cluster states with a two-dimensional topology that are suitable for universal quantum computing and quantum simulation can be readily generated in a deterministic manner, and routes towards fault tolerance via bosonic quantum error correction are known. In this article we propose a complete measurement-based quantum computing architecture for the implementation of a universal set of gates on the recently generated two-dimensional cluster states [M. V. Larsen et al., Science 366, 369 (2019); W. Asavanant et al., Science 366, 373 (2019)]. We analyze the performance of the various quantum gates that are executed in these cluster states as well as in other two-dimensional cluster states (the bilayer-square lattice and quad-rail lattice cluster states [R. N. Alexander et al., Phys. Rev. A 94, 032327 (2016); N. C. Menicucci, Phys. Rev. A 83, 062314 (2011)]) by estimating and minimizing the associated stochastic noise addition as well as the resulting gate error probability. We compare the four different states and find that, although they all allow for universal computation, the quad-rail lattice cluster state performs better than the other three states, which all exhibit similar performance.
\end{abstract}

DOI: 10.1103/PhysRevA.102.042608

\section{INTRODUCTION}

Measurement-based quantum computation (QC) [1] on continuous-variable (CV) cluster states [2,3], also known as cluster state computation, shows great potential for scalable quantum information processing. This is due to the simplicity of generating a deterministic and scalable cluster state resource and the efficiency by which Gaussian gates can be implemented with high-efficiency homodyne detection as already experimentally demonstrated on few-mode cluster states [4-8]. The generation of large one-dimensional (1D) cluster states was realized several years ago [9-11], but for $\mathrm{QC}$ at least two dimensions are required, one for encoding and another for computation. There are multiple feasible proposals for the generation of 2D cluster states [12-16], and recently two different $2 \mathrm{D}$ cluster states were experimentally realized $[17,18]$. A natural question is then how the different $2 \mathrm{D}$ cluster states compare with regard to their suitability for QC.

Computation schemes for some of the popular 2D cluster states already exist $[12,16,19,20]$. Here we summarize these schemes and propose alternative computation schemes for the recently experimentally realized states. Since physical CV cluster states always include noise due to finite squeezing, we furthermore perform a noise analysis of the discussed computation schemes. While similar noise analyses have been done for the 1D dual-rail wire cluster state [21] and the regular 2D square lattice cluster state [22], such analysis on exper-

\footnotetext{
*mivila@fysik.dtu.dk

†ulrik.andersen@fysik.dtu.dk
}

imentally feasible $2 \mathrm{D}$ cluster states has not yet been carried out. Here we aim to find the best noise performance for QC with the discussed schemes on each cluster state.

As such, this work is partly a review of existing computation schemes, an introduction to alternative computation schemes of experimentally realized cluster states, a detailed noise analysis of QC on the different 2D CV cluster states, and a comparison of these. The paper begins with an introduction to the notation and a review of basic concepts in Sec. II. In Sec. III we introduce an alternative computation scheme for the 2D cluster state experimentally realized by us in [17] and perform a noise analysis of this scheme. In Sec. IV we describe corresponding computation schemes on three other popular 2D cluster states, namely, the quad-rail lattice [19], the bilayer square lattice [12], and the recently generated cluster state by Asavanant $e t$ al. [18]. For each of them, we repeat the same noise analysis as presented in Sec. III. In Sec. V we compare the topology and noise performance of the different cluster states and discuss the requirements for universal QC. Finally, we conclude on the results in Sec. VI. Depending on the reader's motivation and prior knowledge of cluster state computation, the reader may skip sections and jump to that of interest; we have carefully cross-referenced the sections of this paper.

\section{PREREQUISITE}

In this section we review the basic concepts of continuousvariable cluster state quantum computation that we will be using in this work. In case the reader is familiar with these concepts, the section can be skipped. 


\section{A. Definitions}

Throughout the paper we assume that $\hbar=1$ and $[\hat{x}, \hat{p}]=i$ such that the light field amplitude $\hat{x}$ (or position) and phase $\hat{p}$ (or momentum) quadratures can be written as $\hat{x}=(\hat{a}+$ $\left.\hat{a}^{\dagger}\right) / \sqrt{2}$ and $\hat{p}=-i\left(\hat{a}-\hat{a}^{\dagger}\right) / \sqrt{2}$, respectively, where $\hat{a}$ is the annihilation operator. With these definitions, the variance of the vacuum is $\frac{1}{2}$. We will make use of six different unitary operators: the identity operator $\hat{I}$, the phase rotation operator $\hat{R}(\theta)=e^{-i \theta\left(\hat{x}^{2}+\hat{p}^{2}\right) / 2}$ (where $\theta$ is the rotation angle) with the Fourier operator $\hat{F}=\hat{R}(\pi / 2)$ as a special case, the squeezing operator $\hat{S}(s)=e^{i \ln (s)(\hat{x} \hat{p}+\hat{p} \hat{x}) / 2}$ [where $r=-\ln (s)$ is the standard squeezing parameter], the shear operator $\hat{P}(p)=e^{i p \hat{x}^{2} / 2}$ (where $p$ is a shearing parameter), the controlled-Z operator $\hat{C}_{Z}(g)=e^{i g \hat{x} \otimes \hat{x}}$ (where $g$ is the coupling coefficient), and the balanced beam splitter operator $\hat{B}=e^{-i \pi(\hat{x} \otimes \hat{p}-\hat{p} \otimes \hat{x}) / 4}$. Each of these operators is Gaussian and can be described by symplectic matrices representing the evolution of the quadrature operators, arranged in a vector $\left(\hat{x}_{1}, \ldots, \hat{x}_{n}, \hat{p}_{1}, \ldots, \hat{p}_{n}\right)^{T}$ for $n$ modes, in the Heisenberg picture

$$
\begin{aligned}
\mathbf{I} & =\left(\begin{array}{ll}
1 & 0 \\
0 & 1
\end{array}\right), \quad \mathbf{R}=\left(\begin{array}{cc}
\cos \theta & \sin \theta \\
-\sin \theta & \cos \theta
\end{array}\right), \\
\mathbf{S} & =\left(\begin{array}{ll}
\frac{1}{s} & 0 \\
0 & s
\end{array}\right), \quad \mathbf{P}=\left(\begin{array}{ll}
1 & 0 \\
p & 1
\end{array}\right), \\
\mathbf{C}_{\mathbf{Z}} & =\left(\begin{array}{llll}
1 & 0 & 0 & 0 \\
0 & 1 & 0 & 0 \\
0 & g & 1 & 0 \\
g & 0 & 0 & 1
\end{array}\right), \quad \mathbf{B}=\frac{1}{\sqrt{2}}\left(\begin{array}{cccc}
1 & -1 & 0 & 0 \\
1 & 1 & 0 & 0 \\
0 & 0 & 1 & -1 \\
0 & 0 & 1 & 1
\end{array}\right)
\end{aligned}
$$

for $\hat{I}, \hat{R}(\theta), \hat{S}(s), \hat{P}(p), \hat{C}_{Z}(g)$, and $\hat{B}$, respectively.

To allow for quantum error correction, in this paper we consider the encoding of qubits in bosonic modes of computation $\left|\psi_{\text {in }}\right\rangle$. Numerous different qubit encodings have been proposed such as encoding in cat states [23,24] and binomial states [25,26], but here we will consider the efficient Gottesman-Kitaev-Preskill (GKP) encoding [27]. For these codes, a qubit is encoded on a square lattice in phase space in a way that allows for the suppression of relevant errors (such as loss) to a certain extend. To combat residual qubit errors, the GKP code must be concatenated with another qubit error-correction code such as the seven-qubit Steane code [28] or Knill's $C_{4} / C_{6}$ code [29]. GKP encoding is the only known bosonic code for which a universal Gaussian gate set allows logic Clifford computation and error correction of encoded qubits. A logic single-mode Clifford gate set is realized by the Gaussian gate set $\{\hat{I}, \hat{F}, \hat{P}(1)\}$ together with displacements, while two-mode gates are enabled by the $\hat{C}_{Z}(1)$ gate. A non-Clifford gate completes the universal encoded qubit gate set. While the non-Clifford gate requires challenging nonGaussian transformations, practical proposals do exist, which are discussed further in Sec. V B. Here in Sec. II, as well as in Secs. III and IV, we will focus on the implementations of the Gaussian gates $\hat{I}, \hat{F}, \hat{P}(1)$, and $\hat{C}_{Z}(1)$.

Gaussian gates are implemented on a cluster state by quadrature measurement of each mode in different bases rotated by $\theta$ with respect to the $\hat{x}$ quadrature, i.e., measuring $\hat{x}(\theta)=\hat{x} \cos \theta+\hat{p} \sin \theta$. In this paper we use the Heisenberg

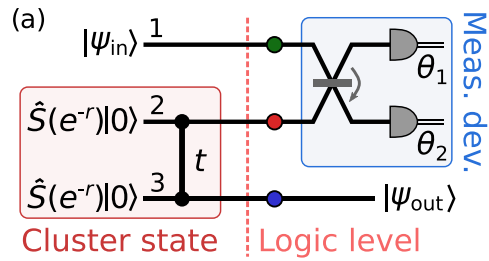

(b)

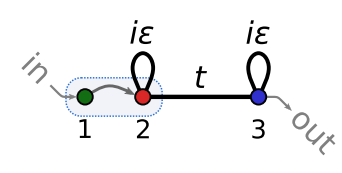

FIG. 1. (a) Generalized teleportation circuit implementing a single-mode Gaussian operation on the input state $\left|\psi_{\text {in }}\right\rangle$ by joint measurement of the input state with one mode of an ancillary approximate cluster state using a two-mode measurement device. The measurement device consists of a beam splitter, with the arrow pointing from the first to the second mode in Eq. (1), followed by homodyne detection measuring in bases $\hat{x}_{i}\left(\theta_{i}\right)=\hat{x}_{i} \cos \theta_{i}+\hat{p}_{i} \sin \theta_{i}$. (b) Short graphical notation of the circuit in (a) used in this paper, with the nodes representing the modes in (a). Here $\varepsilon=e^{-2 r}$. The node colors have no physical meaning and are only used to identify the modes in (a). The graphical notation can be thought of as a snapshot of the logic level in (a) where the computation takes place.

picture, in which we simulate the evolution of the quadrature operators and where the noise contributions simply appear as additive Gaussian noise terms. In the following we consider the generalized teleportation circuit as an example, which as well plays an important role in the quantum computation schemes presented in Secs. III and IV.

\section{B. Generalized teleportation}

An arbitrary Gaussian transformation on a single bosonic mode can be realized by means of the generalized teleportation circuit, as diagrammatically depicted in Fig. 1(a). Here the term generalized teleportation is in terms of the generalized measurement bases, where different gates are implemented on a teleported state depending on the basis setting. Generalized teleportation consists of an input state, an entangled multimode ancillary state, and a measurement device. In conventional single-mode teleportation, the ancillary entangled state is a two-mode squeezed state [30], while traditionally for measurement-based quantum computing (MBQC) we consider a cluster state. The two-mode squeezed state and two-mode cluster state are equivalent (up to a phase rotation), but since well-developed theoretical tools exist for cluster states in the language of graphical calculus [31], here we focus on cluster states. In practice, the ancillary entangled state is an approximate cluster state composed of finitely momentumsqueezed states that are entangled by a controlled- $Z$ gate of weight $t$ [3]. In Fig. 1(a), the momentum variance is $\varepsilon / 2$, where $\varepsilon=e^{-2 r}$, with $r$ the squeezing parameter. To implement a gate, a joint measurement is performed on the input state and one mode of the cluster state using the measurement device marked in Fig. 1(a) consisting of a beam splitter and two homodyne detectors. The resulting transformation of the quadratures in the Heisenberg picture is

$$
\left(\begin{array}{l}
\hat{x}_{3}^{\prime} \\
\hat{p}_{3}^{\prime}
\end{array}\right)=\mathbf{G}\left(\begin{array}{l}
\hat{x}_{1} \\
\hat{p}_{1}
\end{array}\right)+\mathbf{N}\left(\begin{array}{l}
\hat{p}_{2} \\
\hat{p}_{3}
\end{array}\right)+\mathbf{D}\left(\begin{array}{l}
m_{1} \\
m_{2}
\end{array}\right) .
$$

Here the first term represents the implemented Gaussian gate with $\mathbf{G}$ corresponding to the gate symplectic matrix. The second term, with $\mathbf{N}$ being a matrix, represents noise added 
to the quadratures due to finite squeezing in the cluster state; it vanishes in the infinite squeezing limit as $\left\langle\hat{p}_{2,3}\right\rangle=0$ and $\operatorname{Var}\left(\hat{p}_{2,3}\right)=e^{-2 r} / 2 \rightarrow 0$ for $r \rightarrow \infty$. This term represents the gate noise. The last term, with $\mathbf{D}$ being a matrix, is the computational by-product in the form of a displacement, where $m_{1}$ and $m_{2}$ are the measurement outcomes of modes 1 and 2, respectively. The matrices $\mathbf{G}, \mathbf{N}$, and $\mathbf{D}$ are each described in detail in the following sections.

When considering multimode computing schemes with large cluster states, the circuit model in Fig. 1(a) becomes tedious. Instead, it is customary to use graphical notation as illustrated in Fig. 1(b), where the cluster state is represented by its corresponding graph with imaginary self-loops indicating the finite squeezing of the cluster state modes [31], the beam splitter of the measurement device is represented by an arrow, and the input state is represented by a free node. For the schemes presented in this work, we assume that all cluster state modes are equally squeezed. Hence, we will omit the identical $i \varepsilon$ self-loops on the cluster state nodes; they are always there and only vanish in the nonphysical infinite squeezing limit. Finally, we will define the logic level as being the level in the circuit diagram where the computation takes place, i.e., after the cluster state generation where the input state appears and before the measurement device for computation. The logic level is marked in Fig. 1(a), and the graphical notation in Fig. 1(b) is a snapshot of this logic level with the arrow indicating the subsequent beam-splitter operation of the measurement device. An alternative formulation is to use macronodes as in [12,21], where, instead of joint measurements of localized modes in the logic level, one considers single-mode measurements of distributed modes. In Fig. 1(a) this macronode formulation corresponds to locating the logic level right after the beam-splitter transformation and keeping in mind that in the logic level the mode under computation is distributed between modes 1 and 2 as $\left(\hat{a}_{1}+\hat{a}_{2}\right) / \sqrt{2}$.

In the following we describe each term of Eq. (2) in more detail.

\section{Gate}

The gate implemented in Eq. (2) depends on the measurement bases of the two quadrature measurements (defined as $\theta_{1}$ and $\theta_{2}$ ) as

$$
\mathbf{G}=\frac{1}{\sin \theta_{-}}\left(\begin{array}{cc}
\frac{1}{t} \cos \theta_{+}+\frac{1}{t} \cos \theta_{-} & \frac{1}{t} \sin \theta_{+} \\
-t \sin \theta_{+} & t \cos \theta_{+}-t \cos \theta_{-}
\end{array}\right),
$$

where $\theta_{ \pm}=\theta_{1} \pm \theta_{2}$, and corresponds to the operation [32]

$$
\hat{S}(t) \hat{R}\left(\frac{\theta_{+}}{2}\right) \hat{S}\left(\tan \frac{\theta_{-}}{2}\right) \hat{R}\left(\frac{\theta_{+}}{2}\right) .
$$

By implementing such an operation twice, corresponding to two consecutive runs of the teleportation circuit in Fig. 1(a), it is possible to induce an arbitrary single-mode Gaussian transformation [21] $\mathbf{U}(\hat{x}, \hat{p})^{T}+\mathbf{c}$, where $\mathbf{U}=\mathbf{G}_{2} \mathbf{G}_{1}$ while the displacement $\mathbf{c}$ is ubiquitous and simply implemented by shifting the measurement result and updating the bases of the subsequent measurements [2,3]. However, in the following we consider only the subset of single-mode Gaussian transformation that is required for GKP state computation, namely, the set $\{\hat{I}, \hat{F}, \hat{P}(1)\}$.
The identity operator $\hat{I}$ can be executed in a single computation step of the teleportation circuit in Fig. 1(a) by choosing

$$
\left(\theta_{+}, \theta_{-}\right)_{I}=(0,2 \arctan 1 / t),
$$

as easily seen from Eq. (3). For the Fourier gate $\hat{F}$ and the $\hat{P}(1)$ gate two computation steps are necessary: If we let the output state $|\psi\rangle_{\text {out }}$ of the first computation step in Fig. 1(a), with bases $\left(\theta_{+1}, \theta_{-1}\right)$, be the input state on a second identical circuit, but with bases $\left(\theta_{+2}, \theta_{-2}\right)$, the gate

$$
\begin{aligned}
\hat{S}(t) \hat{R} & \left(\frac{\theta_{+2}}{2}\right) \hat{S}\left(\tan \frac{\theta_{-2}}{2}\right) \hat{R}\left(\frac{\theta_{+2}}{2}\right) \\
& \times \hat{S}(t) \hat{R}\left(\frac{\theta_{+1}}{2}\right) \hat{S}\left(\tan \frac{\theta_{-1}}{2}\right) \hat{R}\left(\frac{\theta_{+1}}{2}\right)
\end{aligned}
$$

is implemented. Choosing

$$
\left(\theta_{+1}, \theta_{-1}, \theta_{+2}, \theta_{-2}\right)_{F}=\left(\frac{\pi}{2}, \frac{\pi}{2}, 0,2 \arctan \frac{1}{t^{2}}\right)
$$

implements the $\hat{F}$ gate, while

$$
\left(\theta_{+1}, \theta_{-1}, \theta_{+2}, \theta_{-2}\right)_{P}=\left(\arctan 2,-\arctan 2, \frac{\pi}{2}, \frac{\pi}{2}\right)
$$

implements the $\hat{P}(1)$ gate. To implement the two-mode $\hat{C}_{Z}$ gate a scheme with at least two input modes is required. This will be discussed in Secs. III and IV, where we find that also two teleportation steps are necessary.

\section{Gate noise}

The second term in Eq. (2) represents the gate noise and is governed by the matrix

$$
\mathbf{N}=\left(\begin{array}{cc}
-\frac{1}{t} & 0 \\
0 & 1
\end{array}\right),
$$

which is independent of the measurement bases. However, for gates realized in two steps, such as the $\hat{F}$ and $\hat{P}(1)$ gates described above, the gate noise of the first step enters the gate of the second step, leading to the final gate noise that depends on the bases of the second computation step. For two concatenated circuits of the type in Fig. 1(a), with the cluster state of the second circuit being denoted by modes 4 and 5 , the combined gate noise becomes

$$
\mathbf{G}_{2} \mathbf{N}_{1}\left(\begin{array}{c}
\hat{p}_{2} \\
\hat{p}_{3}
\end{array}\right)+\mathbf{N}_{2}\left(\begin{array}{l}
\hat{p}_{4} \\
\hat{p}_{5}
\end{array}\right) \equiv \mathbf{N}\left(\begin{array}{l}
\hat{p}_{2} \\
\hat{p}_{3} \\
\hat{p}_{4} \\
\hat{p}_{5}
\end{array}\right),
$$

where $\mathbf{N}_{1}\left(\hat{p}_{2}, \hat{p}_{3}\right)^{T}$ is the gate noise of the first step, and $\mathbf{G}_{2}$ and $\mathbf{N}_{2}\left(\hat{p}_{4}, \hat{p}_{5}\right)^{T}$ are the gate symplectic matrix and gate noise of the second step, respectively. Here the combined gate noise matrix $\mathbf{N}$ is a $2 \times 4$ matrix. Note that in Secs. III and IV, $\mathbf{N}$ is in general the combined gate noise matrix of an implemented gate in one or more teleportation steps, with the number of columns equal to the number of quadratures in the output mode(s) and the number of rows equal to the number of ancillary cluster state modes involved in the implemented gate. 
Assuming that all cluster state modes in Eq. (4) are equally squeezed, $\operatorname{Var}\left(\hat{p}_{l}\right)=\varepsilon / 2$ for $l=2,3,4,5$, the gate noise variance amounts to $\sum_{j=1}^{4} N_{i j}^{2} \varepsilon / 2$ for $i=1,2$. From this expression, it is clear that the gate noise can be reduced by both increasing the degree of squeezing of the cluster state modes (reducing $\varepsilon / 2$ ) and minimizing the sums $\sum_{j=1}^{4} N_{i j}^{2}$, $i=1,2$. We will refer to these two sums as the quadrature noise factors, one for each quadrature. In the schemes for gate implementation presented in Secs. III and IV, the focus is on optimizing the quadrature noise factors for the gate set $\left\{\hat{I}, \hat{F}, \hat{P}(1), \hat{C}_{Z}(1)\right\}$ in order to minimize the probability of inducing errors on the GKP-encoded qubit as discussed in Sec. II C.

\section{Displacement by-product}

The displacement matrix in Eq. (2) reads

$$
\mathbf{D}=\frac{\sqrt{2}}{\sin \theta_{-}}\left(\begin{array}{cc}
-\frac{1}{t} \cos \theta_{2} & -\frac{1}{t} \cos \theta_{1} \\
t \sin \theta_{2} & t \sin \theta_{1}
\end{array}\right)
$$

and so, since we know the measurement bases $\left(\theta_{1}, \theta_{2}\right)$ together with the measurement outcome $\left(m_{1}, m_{2}\right)$, the amount of displacement of a single computation step is known. By keeping track of the displacements in each computation step, the displacement can be accounted for in the following steps by updating the measurement bases and results (i.e., feed- forward) [3]. When all gates are Gaussian, the displacement by-product can be compensated for in the measurement result of the final output state $\left|\psi_{\text {out }}\right\rangle$, known as Gaussian parallelism $[2]$.

In this work we will ignore the displacement by-product as it has no effect on the gate noise performance. However, for the actual experimental implementations of the schemes discussed in this work, the compensation for the displacement is important.

\section{Wigner function representation}

To understand the effect of the quadrature transformation and gate noise in Eq. (2), it is useful to analyze the generalized teleportation in the Wigner function representation. This does not add anything new, but considering the gate noise from a different perspective helps to understand it. Here the Wigner function of the input state to the teleportation circuit in Fig. 1, $\left|\psi_{\text {in }}\right\rangle_{1} \hat{S}\left(e^{-r}\right)|0\rangle_{2} \hat{S}\left(e^{-r}\right)|0\rangle_{3}$, is

$$
W_{\text {in }}\left(x_{1}, p_{1}\right) G_{1 / \varepsilon}\left(x_{2}\right) G_{\varepsilon}\left(p_{2}\right) G_{1 / \varepsilon}\left(x_{3}\right) G_{\varepsilon}\left(p_{3}\right),
$$

where $G_{\delta}$ is a normalized Gaussian function of variance $\delta / 2$ and $W_{\text {in }}$ is the Wigner function corresponding to $\left|\psi_{\text {in }}\right\rangle_{1}$, but not necessarily of a pure state. After the quadrature transformation in the generalized teleportation circuit and measurement of $\hat{x}_{1}\left(\theta_{1}\right)$ and $\hat{x}_{2}\left(\theta_{2}\right)$, the output Wigner function corresponding to $\left|\psi_{\text {out }}\right\rangle$ on mode 3 in Fig. 1 becomes

$$
W_{\text {out }}\left(x_{3}, p_{3}\right)=\mathcal{N} G_{1 / \varepsilon}\left(x_{3}\right) \int d \eta_{2} G_{\varepsilon}\left(\eta_{2}\right) G_{t^{2} / \varepsilon}\left(p_{3}-\eta_{2}\right) \int d \eta_{1} G_{\varepsilon / t^{2}}\left(\eta_{1}\right) W_{\text {in }}\left(\mathbf{G}^{-1}\left(\begin{array}{c}
x_{3}-d_{x}-\eta_{1} \\
p_{3}-d_{p}-\eta_{2}
\end{array}\right)\right),
$$

where $\mathcal{N}$ is a normalization factor depending on the basis setting $\left(\theta_{1}, \theta_{2}\right)$ and measurement outcome $\left(m_{1}, m_{2}\right)$, and $\left(d_{x}, d_{p}\right)^{T}=\mathbf{D}\left(m_{1}, m_{2}\right)^{T}$ subtracted from the $\left(x_{3}, p_{3}\right)^{T}$ argument in $W_{\text {in }}$ corresponds to the displacement by-product. Here each index in the vector argument of $W_{\text {in }}$ should be understood as the two arguments in $W_{\text {in }}(\cdot, \cdot)$. It is clear from the expression that the input state undergoes an operation of symplectic matrix $\mathbf{G}$ by the transformation of its Wigner function arguments by $\mathbf{G}^{-1}$. This corresponds to the implemented gate. The gate noise $\mathbf{N}\left(\hat{p}_{2}, \hat{p}_{3}\right)^{T}$ with variance $\left(\varepsilon / t^{2}, \varepsilon\right)^{T} / 2$ is seen to become a Gaussian convolution of the Wigner function with corresponding variance after applying the gate $\mathbf{G}$. Finally, the Wigner function is subjected to a Gaussian envelope in both quadratures: one of variance $t^{2} / 2 \varepsilon$ in the $\hat{p}$ quadrature after convolution in the $\hat{x}$ quadrature with $G_{\varepsilon / t^{2}}$, followed by one of variance $1 / 2 \varepsilon$ in the $\hat{x}$ quadrature after convolution in the $\hat{p}$ quadrature with $G_{\varepsilon}$. These envelopes in each quadrature are the result of convolutions in orthogonal quadratures, as the quadratures are related by the Fourier transform: Convoluting a Wigner function in the $\hat{x}$ quadrature with $G_{\delta}$ leads to an envelope of the Wigner function in the $\hat{p}$ quadrature of $G_{1 / \delta}(p)$ and vice versa.

Two limits of Eq. (5) are interesting: In the ideal infinite squeezing limit, the convolution functions $G_{\varepsilon / t^{2}}$ and $G_{\varepsilon}$ become $\delta$ functions, while the Gaussian envelopes become infinitely broad, and so $W_{\text {out }}\left(x_{3}+d_{x}, p_{3}+d_{p}\right)^{T}=$ $W_{\text {in }}\left(\mathbf{G}^{-1}\left(x_{3}, p_{3}\right)^{T}\right)$. In the limit $t=0$, where we expect no information to transfer from $W_{\text {in }}$ to $W_{\text {out }}$, the envelope on $W_{\text {in }}\left(\mathbf{G}^{-1}\left(x_{3}, p_{3}\right)^{T}\right)$ becomes a $\delta$ function in the $\hat{p}$ quadrature, which is then convoluted by $G_{\varepsilon}$, while the $\hat{x}$ quadrature is convoluted by an infinitely broad Gaussian followed by an envelope of $G_{1 / \varepsilon}$. As a result, for $t=0, W_{\text {out }}\left(x_{3}, p_{3}\right)=$ $G_{1 / \varepsilon}\left(x_{3}\right) G_{\varepsilon}\left(p_{3}\right)$, corresponding to the initial input squeezed state in the cluster state, as expected.

\section{Error correction}

It is now clear that cluster state quantum computation will inevitably suffer from gate noise that will accumulate throughout the computation. To avoid noise accumulation, quadrature error correction is required in between every implemented gate. For this purpose, symmetric GKP states are particularly useful, not only as the qubit but also as ancillaries for error correction. GKP states have been thoroughly reviewed in several places [33,34], while here they are reviewed in terms of MBQC focusing on the added gate noise caused by finite squeezing in the cluster states. The GKP state Wigner function $W_{\text {in }}(\mathbf{x})$ [with $\mathbf{x}=\left(x_{1}, \ldots, x_{n}, p_{1}, \ldots, p_{n}\right)^{T}$ for an $n$ mode state] consists of $\delta$ functions arranged on a square lattice in phase space of each mode with a lattice constant of $\sqrt{\pi}$ and its qubit eigenstates of the Pauli- $Z$ and $-X$ operators are $\left|j_{L}\right\rangle_{X, Z}=\sum_{i \in \mathbb{Z}}|(2 i+j) \sqrt{\pi}\rangle_{x, p}$ in the quadrature eigenstate bases $|s\rangle_{x}$ and $|s\rangle_{p}[27]$.

As a result of the execution of an $n$-mode gate $\mathbf{G}$ in one or more computation steps, the gate noise $\mathbf{N}\left(\hat{p}_{c 1}, \hat{p}_{c 2}, \ldots\right)^{T}$ 
(where $p_{c i}$ are ancillary cluster state modes) leads to a broadening of the GKP $\delta$ functions into Gaussian functions of variances in $\sigma^{2}=\operatorname{Var}\left\{\mathbf{N}\left(\hat{p}_{c 1}, \hat{p}_{c 2}, \ldots\right)^{T}\right\}$ in the $2 n$ quadratures $\left(\sigma^{2}\right.$ is a $2 n$ vector). Furthermore, as the ideal GKP encoding with vanishing variance (represented by $\delta$ functions) is nonphysical, we instead consider the physical approximate GKP states in $W_{\text {in }}(\mathbf{x})$ with $\delta$ functions replaced by symmetric Gaussian functions of identical variance, $\delta$, in $\hat{x}$ and $\hat{p}$ quadrature. The quadrature variance of the Gaussian spikes in the approximate GKP state after the implementation of a noisy gate is then

$$
\boldsymbol{\delta}^{\prime}=\operatorname{Var}\left\{\mathbf{G} \hat{\mathbf{x}}_{\boldsymbol{\delta}}\right\}+\boldsymbol{\sigma}^{2},
$$

where $\hat{\mathbf{x}}$ is decomposed into a sum of $\hat{\mathbf{x}}_{\mathbf{0}}+\hat{\mathbf{x}}_{\boldsymbol{\delta}}$, where $\hat{\mathbf{x}}_{\mathbf{0}}$ and $\hat{\mathbf{x}}_{\boldsymbol{\delta}}$ are the centers and variance of the GKP spikes, respectively. Note that for ideal GKP states $\hat{\mathbf{x}}=\hat{\mathbf{x}}_{\mathbf{0}}$. As examples, $\operatorname{Var}\left\{\mathbf{G} \mathbf{x}_{\delta}\right\}=(\delta, \delta)^{T}$ for the $\hat{I}$ and $\hat{F}$ gates, $\operatorname{Var}\left\{\mathbf{G x}_{\delta}\right\}=$ $(\delta, 2 \delta)^{T}$ for the $\hat{P}(1)$ gate, and $\operatorname{Var}\left\{\mathbf{G} \mathbf{x}_{\delta}\right\}=(\delta, \delta, 2 \delta, 2 \delta)^{T}$ for the two-mode $\hat{C}_{Z}(1)$ gate.

To avoid gate noise accumulating on the GKP-encoded qubit state, after every implemented $\hat{I}, \hat{F}, \hat{P}(1)$, and $\hat{C}_{Z}(1)$ gate, we measure the quadratures $\hat{x} \bmod \sqrt{\pi}$ and $\hat{p} \bmod \sqrt{\pi}$ using ancillary GKP states and perform quadrature error correction by displacing back the state depending on the measurement outcome

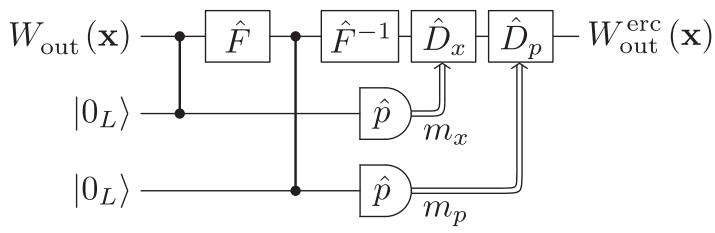

where $\left|0_{L}\right\rangle$ are approximate GKP states with spike variances in both quadratures of $\delta$. Note that while this circuit illustrates the correction algorithm for a single mode of $W_{\text {out }}$, similar circuits are required for each other mode. After the two measurements with outcomes $m_{x}$ and $m_{p}$, the encoded qubit is projected into a "fresh" GKP state, but displaced in $\hat{x}$ and $\hat{p}$ quadratures depending on the values of $m_{x}$ and $m_{p}$ : If $m_{x(p)} \bmod \sqrt{\pi}$ is smaller than $\sqrt{\pi} / 2$, the encoded state is displaced back by $m_{x(p)} \bmod \sqrt{\pi}$ in $\hat{x}(\hat{p})$, while if it is larger than $\sqrt{\pi} / 2$, the encoded states is displaced forward by $\sqrt{\pi}-m_{x(p)} \bmod \sqrt{\pi}$, and so we obtain an error-corrected version of $W_{\text {out }}(\mathbf{x}) \rightarrow W_{\text {out }}^{\text {erc }}(\mathbf{x})$, which is then the input to the next gate. The possible values of $m_{x}$ and $m_{p}$ are Gaussian distributed with variance $\delta_{i}^{\prime}+\delta$, where $\delta_{i}^{\prime}$ in $\delta^{\prime}$ of Eq. (6) is the corresponding spike quadrature variance of the encoded state after gate implementation, $W_{\text {out }}$, and $\delta$ is the spike variance of the $\left|0_{L}\right\rangle$ ancillary states. As a result, for large $\delta_{i}^{\prime}$ and/or $\delta$, there is a risk of measuring a GKP spike closer to its neighboring spikes of the orthogonal qubit state, i.e., outside the bin range $\left[x_{0}-\sqrt{\pi} / 2 ; x_{0}+\sqrt{\pi} / 2\right]$, where $x_{0}$ is the spike center, and thereby inducing a qubit error when "correcting" the state by displacing it in the wrong direction. The combined probability of displacing an $n$-mode encoded state with $2 n$ quadrature corrections in the wrong direction is shown to be [22]

$$
P_{\mathrm{err}}\left(\boldsymbol{\delta}^{\prime}, \delta\right)=1-\prod_{i=1}^{2 n} \operatorname{erf}\left(\frac{\sqrt{\pi}}{2 \sqrt{2\left(\delta_{i}^{\prime}+\delta\right)}}\right),
$$

where each factor in the product term is the probability of a successful quadrature correction. It is important to mention that $P_{\text {err }}$ is not a true qubit error probability, as it does not account for the probability of measuring a spike at its next-nearest-neighbor bin range $\left[x_{0} \pm 3 \sqrt{\pi} / 2 ; x_{0} \pm 5 \sqrt{\pi} / 2\right]$, which leads to a $2 \sqrt{\pi}$ displacement of the GKP state when corrected, and thereby not a qubit error, although it is an error. This leads to, for example, $P_{\text {err }} \rightarrow 1$ for large $\delta_{i}^{\prime}+\delta$, while the actual error probability should be $\frac{1}{2}$. Furthermore, Eq. (8) does not account for the overall envelope on the spikes of the GKP state and for the fact that the error probability is qubit dependent: Displacing the $\hat{p}$ quadrature by $\sqrt{\pi}$ leads to an error on $\left|+_{L}\right\rangle$, but no error on $\left|0_{L}\right\rangle$. Therefore, for a true estimation of the qubit error probability, we need to take these effects into account. However, despite these issues, in this work (as in [22]) we will use $P_{\text {err }}$ as a figure of merit as it constitutes a good approximation to the actual error probability for reasonably large squeezing levels in which $\delta_{i}^{\prime}+\delta$ is small enough for $2 \sqrt{\pi}$ (or larger) displacements to be neglected during quadrature corrections.

Since the two-mode $\hat{C}_{Z}(1)$ gate requires four quadrature corrections, while the $\hat{I}, \hat{F}$, and $\hat{P}(1)$ gates only require two, the error probability after the $\hat{C}_{Z}(1)$ gate is in general larger. In the schemes presented in Secs. III and IV, when possible, we search for a basis setting for the $\hat{C}_{Z}(1)$ gate that minimizes $P_{\text {err. }}$.

\section{DOUBLE-BILAYER SQUARE LATTICE}

Having discussed the general concept of $\mathrm{CV}$ quantum computation and the associated error analysis, we are now equipped with the relevant tools to rigorously analyze the performance of cluster state computation based on different types of cluster states. In this section we will consider the double-bilayer square lattice (DBSL) cluster state, while in Sec. IV we will consider three other known clusters states.

The cylindrical 2D cluster state produced in Ref. [17] can be straightforwardly projected into a universal DBSL cluster which will be analyzed in the following. The cylindrical DBSL cluster state with a 2D topology of Ref. [17] (corresponding to a cylindrical $\mathcal{H}$-graph state) was generated by "coiling up" a 1D cluster state (a dual-rail wire [9]) of temporal mode duration $\tau$ using an $N \tau$ long delay line and interfering it with itself; the generation setup is summarized in Fig. 2(a). As this $\mathcal{H}$-graph state is self-inverse and bipartite for even $N$, it is transformed into a DBSL cluster state through $\pi / 4$ phase rotations of all modes. Since this transformation simply corresponds to a redefinition of the quadratures, the DBSL $\mathcal{H}$-graph state and the corresponding cluster state are equivalent $[17,31]$. In the following we will therefore only consider the DBSL cluster state.

It is also important to note that in Ref. [17] it was shown that the DBSL cluster state can be projected into a regular square lattice cluster state, which is known to be a universal resource for quantum computing. However, due to the resulting low edge weights of this square lattice, this approach is rather inefficient and leads to unnecessary large gate noise. In the following we present a more efficient computation scheme of the DBSL and quantify it by a gate noise analysis. 

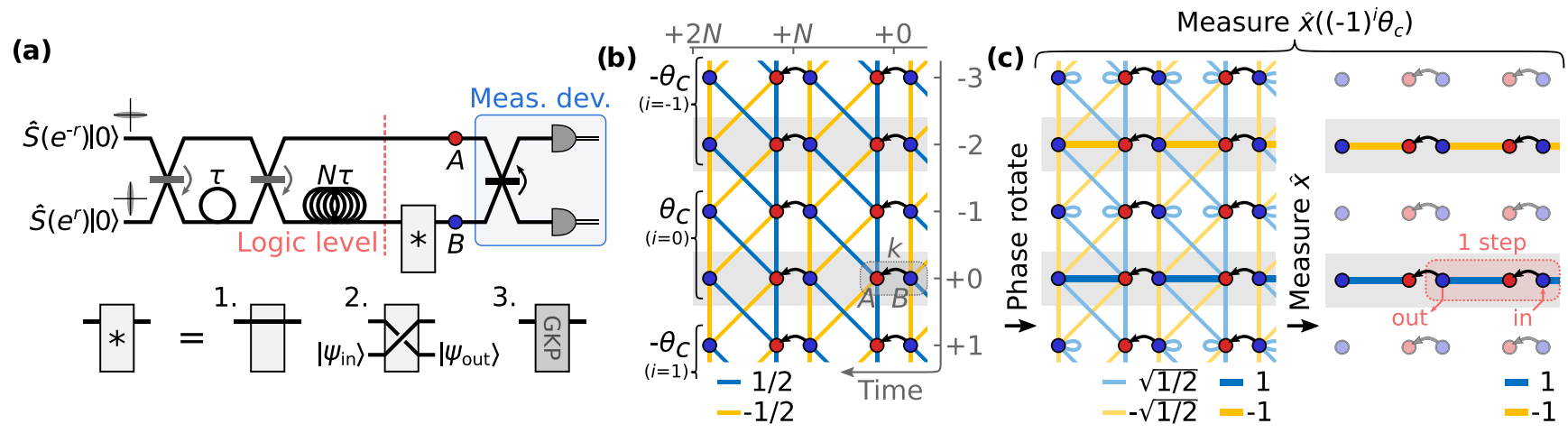

FIG. 2. (a) Temporal encoded DBSL cluster state generation and computation setup. While the DBSL cluster state is generated after the third beam splitter, for computation we consider the cluster state in the marked logic level and the third beam splitter as part of a joint measurement device for computation. The cluster state temporal mode duration $\tau$ is defined by the short delay line. The device marked by an asterisk is an identity gate when implementing gates (1), an optical switch when switching in a state for computation (2), or the circuit in Eq. (7) for correcting gate noise after each implemented gate (3). (b) Cluster state in the logic level. A dual-rail wire is coiled up by the $N \tau$ long delay, leading to a cylinder with $N$ temporal modes in the circumference; the temporal mode indices are marked on the time axis. Computation is performed in wires including the modes in the marked gray areas, while modes in between the gray areas are control modes. Measuring the control modes in an alternating basis of $(-1)^{i} \theta_{c}$ induces edges between the wire modes as shown in (c), allowing single-mode computation in each $N / 2$ wire with one computation step marked with a red area of dotted edge. Measuring a control mode in a different basis creates coupling between the two neighboring wires as shown in Fig. 3, allowing multimode gates. The edge weights shown here are in the limit of infinite squeezing and $\theta_{c}=\pi / 4$. For finite squeezing the edge weights are multiplied by tanh $2 r$ while self-loops of $i$ sech $2 r$ are present on each cluster state mode. For easy comparison, the experimental setup, logic cluster state, and its projection into wires are shown in Appendix C together with the schemes considered in Sec. IV.

\section{A. Efficient computation scheme}

Similar to the generalized teleportation scheme in Sec. II, we define a multimode measurement device that includes the third beam splitter as marked in Fig. 2(a). The resulting logic level is located just before the measurement device, where the generated 1D cluster state is coiled up, but not yet interfered with itself. A section of the cylindrical coiled up 1D cluster state at the logic level is shown in Fig. 2(b). Here the horizontal direction follows the cluster state cylinder axis, while the vertical direction corresponds to the circumference of the cylinder whose size is limited by the long delay line to $N$ temporal modes.

In the following we assume that the cylindrical cluster state has an even number of temporal modes in the circumference ( $N$ is an even number, which is necessary for the generated $\mathcal{H}$ graph state to be bipartite), each with a temporal mode index $k$. Every second temporal mode $(k+2 i$ for $i \in \mathbb{Z})$ forms wires for computation along the cylinder [shaded area in Fig. 2(b)], while the remaining temporal modes $(k+2 i-1$ for $i \in \mathbb{Z})$ are control modes that are used to control couplings between wires. In this way we have $N / 2$ wires, and thereby $N / 2$ modes for computation. We will further assume that the number of wires is even, i.e., $N / 2$ being an even number. As an example, the experimental realization of the DBSL in [17] had $N=12$ leading to six wires. Using an optical switch in the lower spatial mode at point $B$ in the logic level in Fig. 2(a), an input state can be switched into the circuit. It corresponds to adding input states to the blue (marked by $B$ ) nodes in Fig. 2(b). Optical switches have previously been demonstrated in quantum settings [35,36].

By inducing certain phase rotations $(-1)^{i} \theta_{c}$ of the control modes it is possible to create new edges along the wires as illustrated in Fig. 2(c) [31]. If these phase rotations are followed by measurement of the control modes in the $\hat{x}$ basis, the modes and their edges are "deleted" and we are consequently left with $N / 2$ parallel wires suitable for single-mode computation of $N / 2$ modes. It is also worth noting that this combination of phase rotation and $\hat{x}$ measurement corresponds to measuring the quadrature $\hat{x}\left([-1]^{i} \theta_{c}\right)$ on each control mode individually,

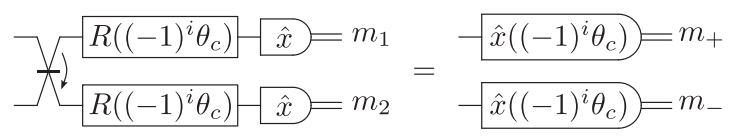

where the beam splitter on the left-hand side is the beam splitter of the measurement device and $m_{ \pm}=\left(m_{1} \pm m_{2}\right) / \sqrt{2}$. As an example, we may consider the case of infinite squeezing as pictured in Fig. 2(c). Here the edge weights of the wires tend towards the optimal values of \pm 1 (where the sign alternates between neighboring wires) by choosing $\theta_{c}=\pi / 4$. For finite, thus practical, squeezing levels, the induced wire edge weight is lower, while $\theta_{c}$ may be optimized for minimizing the gate noise. For simplicity, in the computation scheme presented here, we keep $\theta_{c}=\pi / 4$ for all squeezing levels, while in Sec. III C we discuss the effect of varying $\theta_{c}$.

The projected wires in Fig. 2(c) are now suitable for single-mode Gaussian computation: One computation step (one horizontal time step from temporal mode $k$ to $k+N$ ) corresponds to the generalized teleportation circuit in Sec. II B with an input from the previous computation step, or switched into the cluster using an optical switch as previously mentioned. Similar to the generalized teleportation, the resulting operation of one single-mode computation step on a wire is

$$
\hat{S}\left([-1]^{i} 4 t^{2}\right) \hat{R}\left(\frac{\theta_{+}}{2}\right) \hat{S}\left(\tan \frac{\theta_{-}}{2}\right) \hat{R}\left(\frac{\theta_{+}}{2}\right),
$$


(a)

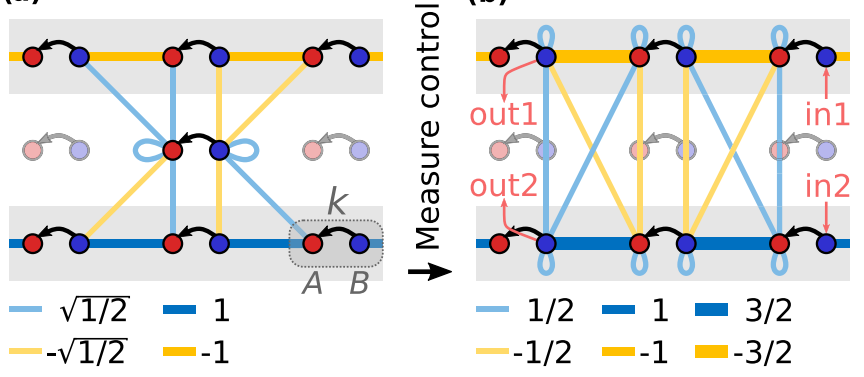

FIG. 3. (a) Logic cluster state after measuring all temporal control modes in Fig. 2(c) except control modes in temporal mode $k+N-1$. (b) Cluster state after further phase rotation and measurement of the remaining two control modes in (a), leading to direct edges between the neighboring two wires. The edge weights shown here are for the case of infinite squeezing, $\theta_{c}=\pi / 4$, and the central control modes further phase rotated by $\arctan \frac{1}{2}$, i.e., $g=1$ in Eq. (11).

where $\theta_{ \pm}=\theta_{B k} \pm \theta_{A k}$ and $t$ is the absolute edge weight in the logic dual-rail wire cluster state in Fig. 2(b), which equals $\frac{1}{2}$ in the infinite squeezing limit; the case of finite squeezing is discussed in Sec. III B. For derivation of (10) see Appendix A. The negative edge weight on every second wire (uneven $i$ ) leads to a $\pi$ phase rotation in each computation step which then cancels out in every second step or can be compensated for in the required basis setting for the desired gate. As for the generalized teleportation, any Gaussian single-mode gate can be implemented in two steps.

Now let us discuss how a two-mode gate can be implemented by coupling two neighboring wires. In Fig. 3(a) all control modes except one have been measured in the basis $(-1)^{i} \theta_{c}=(-1)^{i} \pi / 4$ in order to separate wires as described above; the remaining two central control modes in Fig. 3(a) have only been phase shifted by $\pi / 4$, but not measured. Phase rotating these remaining control modes further before measurements (i.e., measuring them in another basis than the neighboring control modes) leads to coupling between the two neighboring wires which is seen as direct edges in Fig. 3(b). In this way, by controlling the measurement bases of a temporal control mode, together with the measurement bases of its neighboring wires, a desired two-mode gate can be implemented. As an example, in the infinite squeezing limit of Fig. 3, the base setting

$$
\theta \equiv\left(\begin{array}{c}
\theta_{A k-2} \\
\theta_{B k-2} \\
\theta_{A k} \\
\theta_{B k} \\
\theta_{A k+N-2} \\
\theta_{B k+N-2} \\
\theta_{A k+N-1} \\
\theta_{B k+N-1} \\
\theta_{A k+N} \\
\theta_{B k+N}
\end{array}\right)=\left(\begin{array}{c}
(-1)^{i} 3 \pi / 8 \\
-(-1)^{i} \pi / 8 \\
(-1)^{i} 3 \pi / 8 \\
-(-1)^{i} \pi / 8 \\
(-1)^{i} \pi / 4-\arctan (g / 2) \\
-(-1)^{i} \pi / 4 \\
(-1)^{i} \pi / 4+\arctan (g / 2) \\
(-1)^{i} \pi / 4+\arctan (g / 2) \\
(-1)^{i} \pi / 4-\arctan (g / 2) \\
-(-1)^{i} \pi / 4
\end{array}\right)
$$

leads to an implementation of the gate $[\hat{R}(\pi / 4) \otimes$ $\hat{R}(\pi / 4)] \hat{C}_{Z}(g)$ between the blue input modes (marked by $B$ ) in temporal modes $k-2$ and $k$, where $\hat{R}(\pi / 4) \otimes \hat{R}(\pi / 4)$ can be compensated for with the following single-mode gates.

In summary, we have now shown that a universal Gaussian gate set can be efficiently implemented on a DBSL cluster state: Single-mode gates can be realized along parallel wires in the cluster, while the two-mode controlled- $Z$ gate can be realized between neighboring wires.

\section{B. Gate noise analysis}

As mentioned previously, if the squeezed states used to construct the cluster state are infinitely squeezed, the gates will be realized perfectly without noise addition, thus without adding any processing errors. However, in a realistic setting, the degree of squeezing is finite, which inevitably will result in processing noise. In the following we will be analyzing the impact it has when using the DBSL for computation.

Assuming that the two squeezed input states of the circuit in Fig. 2(a) have squeezed variances of $e^{-2 r}$ in the $\hat{x}$ or $\hat{p}$ quadrature, the edge weights and self-loops of the coiled up $1 \mathrm{D}$ cluster state at the logic level become $\pm t= \pm \tanh (2 r) / 2$ and $i \varepsilon=i \operatorname{sech} 2 r$, respectively [17,31]. Note that the existence of self-loops is a result of the finite input squeezing, while $\sqrt{\varepsilon}$ can be considered as the effective momentum squeezing in the cluster state modes.

The finite squeezing leads to two effects: gate noise appearing in each computation step and distortion of the implemented gate. As seen in Eq. (10), for single-mode gates the distortion is caused by an additional squeezing transformation $\hat{S}\left([-1]^{i} \tanh ^{2} 2 r\right)$ on the output of each computation step. However, as for generalized teleportation, the unwanted squeezing transformation can be compensated for simply by tuning the basis settings. The gate noise [introduced in Sec. II B and corresponding to the second term of Eq. (2)] of one single-mode computation step from temporal mode $k$ to $k+N$ (derived in Appendix A) is represented by the quadratures

$$
\mathbf{N}\left(\hat{p}_{A k-1}, \hat{p}_{A k}, \hat{p}_{A k+1}, \hat{p}_{B k+N-1}, \hat{p}_{B k+N}, \hat{p}_{B k+N+1}\right)^{T},
$$

with

$$
\mathbf{N}=\left(\begin{array}{cccccc}
\frac{1}{4 t} & \frac{-1}{4 t^{2}} & \frac{-1}{4 t} & \frac{1}{4 t} & 0 & \frac{1}{4 t} \\
t & 0 & t & t & 1 & -t
\end{array}\right)
$$

leading to quadrature noise factors (introduced in Sec. II B) of

$$
\begin{aligned}
& N_{x}=\sum_{j} N_{1 j}^{2}=\frac{1}{\tanh ^{4} 2 r}+\frac{1}{\tanh ^{2} 2 r}, \\
& N_{p}=\sum_{j} N_{2 j}^{2}=\tanh ^{2}(2 r)+1
\end{aligned}
$$

in $\hat{x}$ and $\hat{p}$, respectively.

To avoid accumulating gate noise during computation, we consider the usage of GKP-encoded qubit states, in which the gate noise is translated into qubit errors by quadrature corrections after each implemented gate using auxiliary GKP states as described in Sec. II C. To prevent erroneous computation, the qubits may then be error corrected by including a qubit error-correction scheme in the computation. Within the 
GKP-encoded qubit subspace, a logic complete Clifford gate set is realized by the Gaussian gate set $\left\{\hat{I}, \hat{F}, \hat{P}(1), \hat{C}_{Z}(1)\right\}$ on the bosonic modes. We therefore only consider the implementation of this gate set in the noisy cluster state. An additional non-Clifford gate in the GKP qubit subspace completes the gate set for universal qubit computation and is further discussed in Sec. V B.

Similar to the generalized teleportation circuit in Sec. II B [Eq. (3)] but by replacing the edge weights $t$ with $(-1)^{i} \tanh ^{2} 2 r$, the single-mode gate $\hat{I}$ is implemented from temporal mode $k$ to $k+N$ with the basis setting

$$
\left(\begin{array}{l}
\theta_{+} \\
\theta_{-}
\end{array}\right)_{I}=\left(\begin{array}{c}
0 \\
(-1)^{i} 2 \arctan \left(\tanh ^{-2} 2 r\right)
\end{array}\right),
$$

where $\theta_{ \pm}=\theta_{B k} \pm \theta_{A k}$ and with gate noise variances of $N_{x} \varepsilon / 2$ and $N_{p} \varepsilon / 2$ in $\hat{x}$ and $\hat{p}$ quadratures, respectively. The $\hat{F}$ and $\hat{P}(1)$ gates are implemented in two computation steps from mode $k$ to $k+2 N$ : Choosing

$$
\left(\begin{array}{c}
\theta_{+1} \\
\theta_{-1} \\
\theta_{+2} \\
\theta_{-2}
\end{array}\right)_{F}=\left(\begin{array}{c}
\pi / 2 \\
\pi / 2 \\
0 \\
2 \arctan \left(\tanh ^{-4} 2 r\right)
\end{array}\right)
$$

implements $\hat{F}$ with equal gate noise variance of $\left(N_{x}+N_{p}\right) \varepsilon / 2$ in $\hat{x}$ and $\hat{p}$, while

$$
\left(\begin{array}{l}
\theta_{+1} \\
\theta_{-1} \\
\theta_{+2} \\
\theta_{-2}
\end{array}\right)_{P}=\left(\begin{array}{c}
\arctan 2 \\
-\arctan 2 \\
\pi / 2 \\
\pi / 2
\end{array}\right)
$$

implements $\hat{P}(1)$ with gate noise variances of $2 N_{x} \varepsilon / 2$ and $2 N_{p} \varepsilon / 2$ in $\hat{x}$ and $\hat{p}$, respectively. Here $\theta_{ \pm 1}=\theta_{B k} \pm \theta_{A k}$ and $\theta_{ \pm 2}=\theta_{B k+N} \pm \theta_{A k+N}$. For the two-mode $\hat{C}_{Z}$ gate, the gate distortion due to finite squeezing, as well as how it is compensated for, is less trivial. In the following we search for basis settings that compensate for finite squeezing and optimize the gate noise in order to minimize the error probability of the encoded qubit after quadrature corrections.

The GKP quadrature corrections can be realized by implementing the circuit in Eq. (7) at mode $B$ in the logic level in Fig. 2 where the processed state is encoded. This may be challenging, as it requires tunable $\hat{C}_{Z}(g)$ coupling strengths with $g=1$ when performing error correction and $g=0$ otherwise. An alternative is to occupy the free wires with ancillary $\left|0_{L}\right\rangle$ GKP states and then implement the required $\hat{C}_{Z}(1)$ gates through measurements. However, with this approach, the error-correcting gate is subjected to the same kind of gate noise that we are trying to correct for in the encoded state. For simplicity, we assume successful implementation of the quadrature correction circuit in Eq. (7) at the logic level using a supply of ancillary GKP states with quadrature symmetric spike variance equal to the variance of the resource squeezing $\delta=e^{-2 r} / 2$.

As discussed in Sec. III A and illustrated in Fig. 3, the $\hat{C}_{Z}(1)$ gate between two wires is implemented in two computation steps, and while staying within the encoded qubit subspace, we are allowed to implement any $\hat{C}_{Z}(1)$ gate with a by-product of gates in $\{\hat{F}, \hat{P}(1)\}$ in order to minimize the resulting GKP-encoded qubit errors. The $\hat{P}(1)$ gate transforms quadratures as $(\hat{x}, \hat{p}) \rightarrow(\hat{x}, \hat{x}+\hat{p})$, which, before adding gate noise, already leads to an increase of the spike variances in the GKP-encoded state as $(\delta, \delta) \rightarrow(\delta, 2 \delta)$, where the first and second indices correspond to variance in the $\hat{x}$ and $\hat{p}$ quadratures, respectively. Thus, adding $\hat{P}(1)$ gates to $\hat{C}_{Z}(1)$ will hardly improve the error probability. On the other hand, the $\hat{F}$ gate transforms the quadratures as $(\hat{x}, \hat{p}) \rightarrow(\hat{p},-\hat{x})$ and the GKP spike variance in each quadrature (before adding gate noise) is unchanged. Hence, we may improve the error probability if we can improve the resulting gate noise by adding $\hat{F}$ gates to the $\hat{C}_{Z}(1)$ gate. We have investigated the gates $\left(\hat{F}^{n} \otimes\right.$ $\left.\hat{F}^{m}\right) \hat{C}_{Z}(1)$ for all $n, m \in\{-1,0,1,2\}$ and find that gates with $n, m= \pm 1$ are optimal. We choose $n=1$ and $m=(-1)^{i}$, where the index $i$ denotes the control modes between the two coupled wires. The improvement on the $\hat{C}_{Z}(1)$ gate noise by adding $\hat{F} \otimes \hat{F}^{ \pm 1}$ may be explained intuitively: The $\hat{F}$ gates rotate the states in computation during the two computation steps implementing $\left(\hat{F} \otimes \hat{F}^{ \pm 1}\right) \hat{C}_{Z}(1)$, which leads to the gate noise being better distributed on the quadratures, similar to the symmetrically distributed gate noise when implementing the single-mode $\hat{F}$ gate as described above. The by-product of $\hat{F} \otimes \hat{F}^{ \pm 1}$ can then be compensated for by applying the associated single-mode gates after GKP error correction. In the following we first consider the case for even $i$ and to shorten the notation we write $\hat{F} \hat{F} \hat{C}_{Z}$ where the tensor product and $\hat{C}_{Z}$ weight have been ignored.

To implement the $\hat{F} \hat{F} \hat{C}_{Z}$ gate between two neighboring wires as in Fig. 3, we adjust the basis setting $\theta$ in Eq. (11). Using a global search algorithm, we search for $\theta$ minimizing the objective function

$$
f(\theta)=\|\mathbf{G}-\mathbf{T}\|_{1}+w \log _{10} P_{\text {err }}\left(\boldsymbol{\delta}^{\prime}, \delta\right),
$$

where $\mathbf{G}$ and $\mathbf{T}$ are the symplectic matrices of the implemented gate, governed by $\theta$, and the target gate $\hat{F} \hat{F} \hat{C}_{Z}$ (see Appendix A for the procedure of calculating $\mathbf{G}),\|\mathbf{A}\|_{1}=$ $\sum_{i, j}\left|A_{i j}\right|$ is the entrywise matrix 1-norm, and $P_{\text {err }}$ is the error probability in Eq. (8). Here $\delta=e^{-2 r} / 2$ for the ancillary GKP states and $\delta^{\prime}=(2 \delta, 2 \delta, \delta, \delta)^{T}+\sigma^{2}$ for the $\hat{F} \hat{F} \hat{C}_{Z}$ gate with gate noise variance $\boldsymbol{\sigma}^{2}=\left(N_{x 1}, N_{x 2}, N_{p 1}, N_{p 2}\right)^{T} \operatorname{sech}(2 r) / 2$, where $N_{i}$ are basis-dependent quadrature noise factors. The first term of $f(\theta)$ in Eq. (13) is minimized for $\mathbf{G}=\mathbf{T}$ and thus helps us find the basis setting implementing the target gate $\mathbf{T}$. Since multiple solutions $\theta$ leading to $\mathbf{G}=\mathbf{T}$ may exist, we search for a solution that also minimizes the error probability $P_{\text {err }}$, which is the purpose of the second term in Eq. (13). To resolve $P_{\text {err }}$ close to 0 we use the logarithm of $P_{\text {err }}$, while the weight $w$ is varied in the range $10^{-8}-1$ for different resource squeezing in order for the global search algorithm not to favor one term in (13) while ignoring the other term. Finally, the objective function is considered successfully minimized only when the resulting gate is close to the target gate. To check this, we use the condition

$$
\|\mathbf{G}-\mathbf{T}\|_{1}<10^{-5},
$$

with all results not satisfying this condition being discarded. Depending on the global search algorithm used, we are not 

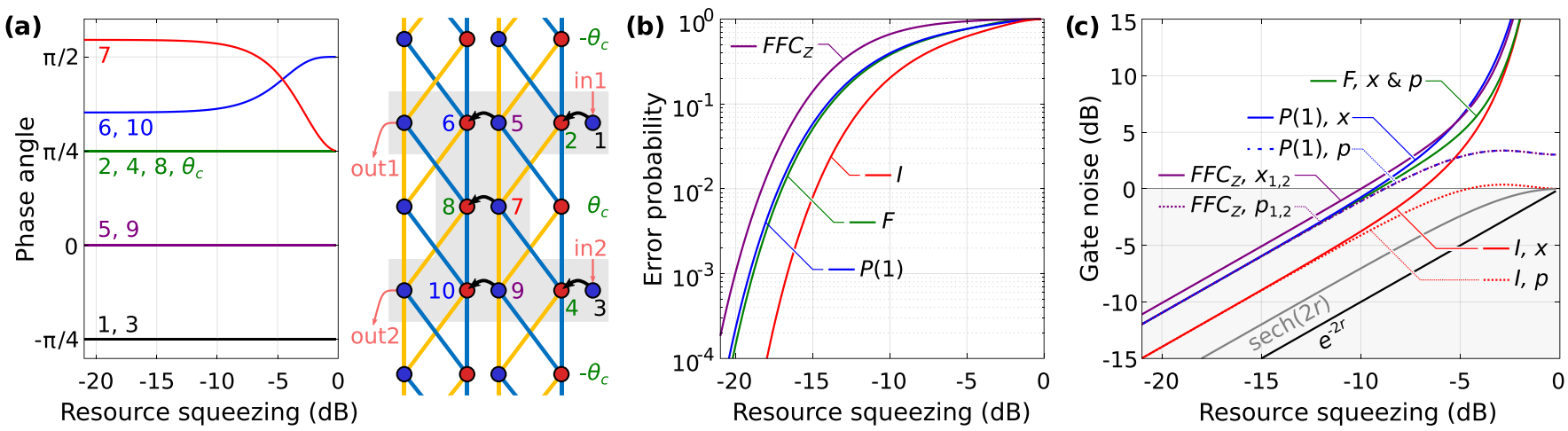

FIG. 4. (a) Basis settings as a function of resource squeezing found by minimizing $f(\theta)$ in Eq. (13) for implementing the $\hat{C}_{Z}(1)$ gate as $(\hat{F} \otimes \hat{F}) \hat{C}_{Z}(1)$ (in short $\hat{F} \hat{F} \hat{C}_{Z}$ ) for even $i$ on the central control modes. The mode numbers are labeled on the graph to the right, where control modes outside the shaded area are measured in the basis $(-1)^{i} \theta_{c}=(-1)^{i} \pi / 4$. For uneven $i,\left(\hat{F} \otimes \hat{F}^{-1}\right) \hat{C}_{Z}(1)$ is implemented with the same gate noise by changing the sign of the bases in modes $3,4,6,7,8$, and 10. (b) Resulting error probabilities of Eq. (8) for the $\hat{I}, \hat{F}$, and $\hat{P}(1)$ gates and the $\left(\hat{F} \otimes \hat{F}^{ \pm 1}\right) \hat{C}_{Z}(1)$ gate implemented with the basis settings in (a). (c) Gate noise responsible for the error probability in (b) together with approximate ancillary GKP states of the $e^{-2 r} / 2$ spike variance. Here the resource variance squeezing $e^{-2 r}$ and effective variance squeezing in the cluster state modes sech $2 r$ are shown as well (the two lower black and gray lines, respectively). The resource squeezing and gate noise in decibel scale are relative to a vacuum variance of $\frac{1}{2}$. Note that the $\hat{p}$-quadrature gate noise of the $\hat{P}(1)$ and $\hat{F} \hat{F} \hat{C}_{Z}$ gates overlap. For easy comparison, the gate noise here is shown in Appendix $\mathrm{C}$ together with the gate noise of the schemes considered in Sec. IV.

guaranteed to find the best basis settings minimizing the error probability. However, repeating the algorithm many times with different $w$ and starting points increases the confidence of the resulting basis settings being optimal.

The resulting bases minimizing the objective function $f(\theta)$ for the $\hat{F} \hat{F} \hat{C}_{Z}$ target gate (with even $i$ for the central control mode) are presented in Fig. 4(a) for different resource squeezing levels as input in Fig. 2(a). In the following we will refer to the mode numbering labeled in Fig. 4(a). According to Eq. (10), with $\left(\theta_{1,3}, \theta_{2,4}\right)=(-\pi / 4, \pi / 4)$ in the first computation step, ignoring the coupling between wires, the input mode is simply teleported to the second computation step with a by-product (in addition to displacement) of $\hat{S}\left(\tanh ^{2} 2 r\right)$. Here control mode 8 is measured in the same $\theta_{c}=\pi / 4$ basis used for separating wires, while control mode 7 is measured in a different basis in order to couple the two wires. With the combined basis setting of modes 5, 6, 7, 9, and 10, the by-product squeezing of the first step is compensated and the $\hat{F} \hat{F} \hat{C}_{Z}$ gate is implemented. Finally, for uneven $i$ on the control modes between the two coupled wires, the $\left(\hat{F} \otimes \hat{F}^{-1}\right) \hat{C}_{Z}(1)$ gate is implemented by changing the sign on modes $3,4,6,7,8$, and 10 . The resulting gate noise and error probability are the same as for $\hat{F} \hat{F} \hat{C}_{Z}$ with even $i$.

After quadrature correction in the GKP scheme the resulting error probability of the above-described basis settings for the $\hat{I}, \hat{F}$, and $\hat{P}(1)$ single-mode gates and the two-mode $\hat{F} \hat{F} \hat{C}_{Z}$ gate are shown in Fig. 4(b). As expected, the error probability is seen to go towards 0 for increasing resource squeezing and towards 1 for vanishing squeezing. Furthermore, the $\hat{F} \hat{F} \hat{C}_{Z}$ gate is seen to have the highest error probability due to four successful quadrature corrections necessary to avoid qubit error, while the $\hat{I}$ gate leads to the lowest error probability as it is implemented in a single computation step. In Sec. V A these error probabilities are compared with error probabilities when using other relevant cluster states and computing schemes.
To gain a better understanding of the error probabilities, we consider the responsible gate noise. The gate noise variance, for the basis settings in Fig. 4(a) and described above, is plotted in Fig. 4(c). In the large squeezing limit, the effective variance squeezing in the cluster state modes of $\operatorname{sech} 2 r$ is a factor of $2(3 \mathrm{~dB})$ larger than the resource variance squeezing of $e^{-2 r}$, which is the cost of preparing the cluster state with off-line squeezing [3]. The $\hat{I}$ gate, implemented in a single computation step, has a gate noise in the range two times higher than the effective squeezing due to $N_{x}, N_{p} \rightarrow 2$ for $r \rightarrow \infty$. The $\hat{F}$ and $\hat{P}(1)$ gates have further gate noise of around a factor 2 , since they are implemented in two computation steps. Finally, the $\hat{F} \hat{F} \hat{C}_{Z}$ gate, also implemented in two computation steps, has similar gate noise, but slightly higher due to the noise of an additional control mode included in the gate to couple two neighboring wires. The gate noise is in general asymmetric in the quadratures (besides for the $\hat{F}$ gate with equal noise factors in the two quadratures), also for the $\hat{F} \hat{F} \hat{C}_{Z}$ gate with optimized basis settings: Since the $P_{\text {err }}$ in Eq. (8) rely on the product of quadrature correction success and because the encoded GKP spike noise is also asymmetric after the $\hat{F} \hat{F} \hat{C}_{Z}$ and $\hat{P}(1)$ gates, the error probability is not necessarily minimum with quadrature symmetric gate noise, and at low squeezing we see the majority of the gate noise in one quadrature. Finally, in the vanishing squeezing limit the gate noise diverges. To understand this, consider the Wigner function transformation of the generalized teleportation in Eq. (5): With the diverging gate noise variance, the Wigner function is convoluted with infinitely broad Gaussian functions in $\hat{x}$ and subjected to corresponding $\delta$-function envelopes in $\hat{p}$, erasing all information of the encoded state. Together with convolutions in the $\hat{p}$ quadrature and corresponding envelopes in the $\hat{x}$ quadrature, the Wigner function is ensured to go towards vacuum for $0-\mathrm{dB}$ resource squeezing. This is further described in Appendix B with the Wigner function transformation of single-mode gates on the DBSL. 

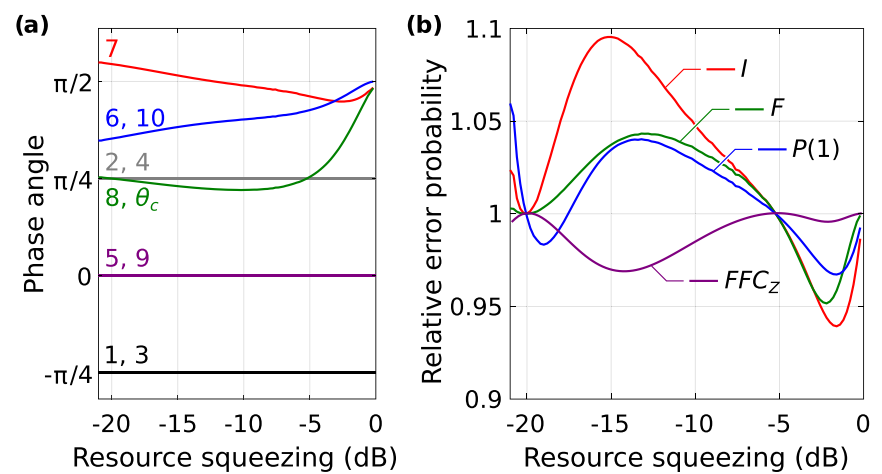

FIG. 5. (a) Basis setting implementing $(\hat{F} \otimes \hat{F}) \hat{C}_{Z}(1)$ (shortened $\hat{F} \hat{F} \hat{C}_{Z}$ ) for even $i$ with variable control basis $\theta_{c}$ minimizing the error probability of Eq. (8), while $\left(\hat{F} \otimes \hat{F}^{-1}\right) \hat{C}_{Z}(1)$ is implemented for uneven $i$ by changing the sign on modes $3,4,6,7,8$, and 10 . Here the mode numbering used is that of Fig. 4(a). (b) Resulting error probabilities using variable $\theta_{c}$ in (a) optimized for $\hat{F} \hat{F} \hat{C}_{Z}$, relative to the corresponding error probabilities in Fig. 4(b) for fixed $\theta_{c}=\pi / 4$.

\section{Variable control mode basis}

For simplicity, so far, we have set the control mode basis to $\theta_{c}=\pi / 4$, which only leads to unity wire edge weight in the infinite squeezing limit. Allowing variable $\theta_{c}$ implements

$$
\hat{S}\left([-1]^{i} 4 t^{2} \tan \theta_{c}\right) \hat{R}\left(\frac{\theta_{+}}{2}\right) \hat{S}\left(\tan \frac{\theta_{-}}{2}\right) \hat{R}\left(\frac{\theta_{+}}{2}\right)
$$

single-mode gates in each computation step with

$$
\mathbf{N}=\left(\begin{array}{cccccc}
\frac{1}{4 t} & \frac{-1}{4 t^{2} \tan \theta_{c}} & \frac{-1}{4 t} & \frac{1}{4 t} & 0 & \frac{1}{4 t} \\
t \tan \theta_{c} & 0 & t \tan \theta_{c} & t \tan \theta_{c} & 1 & -t \tan \theta_{c}
\end{array}\right)
$$

for the gate noise leading to

$$
\begin{aligned}
& N_{x}=\sum_{j} N_{1 j}^{2}=\frac{1}{\tanh ^{4} 2 r \tan ^{2} \theta_{c}}+\frac{1}{\tanh ^{2} 2 r}, \\
& N_{p}=\sum_{j} N_{2 j}^{2}=\tanh ^{2} 2 r \tan ^{2} \theta_{c}+1
\end{aligned}
$$

noise factors. As a result, by varying $\theta_{c}$ we are able to distribute the gate noise between the quadratures in order to minimize the GKP-encoded qubit errors.

To prevent unwanted couplings between wires, $\theta_{c}$ needs to be the same for all gates. With the two-mode $\hat{C}_{Z}(1)$ gate being the gate of largest error probability, we may optimize $\theta_{c}$ to minimize the error probability of the $\left(\hat{F} \otimes \hat{F}^{ \pm 1}\right) \hat{C}_{Z}(1)$ gate. In Fig. 5 optimized basis settings, as well as the corresponding error probabilities relative to the error probabilities for fixed $\theta_{c}=\pi / 4$, are shown as a function of resource squeezing when including $\theta_{c}$ in the objective function in Eq. (13). The error probability for the $\left(\hat{F} \otimes \hat{F}^{ \pm 1}\right) \hat{C}_{Z}(1)$ gate, for which $\theta_{c}$ is optimized, is seen at best to decrease to 0.97 of the error probability with fixed $\theta_{c}$, and thus the gain of variable $\theta_{c}$ is little. Furthermore, since $\theta_{c}$ is only optimized for the $\left(\hat{F} \otimes \hat{F}^{ \pm 1}\right) \hat{C}_{Z}(1)$ gate, for some ranges of resource squeezing, the error probabilities for the $\hat{I}, \hat{F}$, and $\hat{P}(1)$ gates are seen to become worse. In conclusion, there may be a small advantage of optimizing $\theta_{c}$, but this depends on the amount of resource squeezing available and what gates dominate the quantum algorithm to be implemented.

\section{OTHER CLUSTER STATES}

Besides the DBSL, there are three other interesting cluster states with corresponding self-inverse and bipartite $\mathcal{H}$-graph states and thus realizable with off-line squeezing and linear optics: the quad-rail lattice (QRL) [13] with the efficient computation scheme in Ref. [19]; the bilayer square lattice (BSL) $[12,16]$, also with an efficient computation scheme; and the recently demonstrated cluster state by Asavanant et al. [18]. In the following we refer to this last cluster state as the modified bilayer square lattice (MBSL) since computation on this state is similar to computation on the BSL with few modifications. Below we summarize the computation schemes for each cluster state focusing on the $\left\{\hat{I}, \hat{F}, \hat{P}(1), \hat{C}_{Z}(1)\right\}$ gate set which, together with $\sqrt{\pi}$ displacements in $\hat{x}$ and $\hat{p}$ quadratures, constitute a universal Clifford gate set in the GKP-encoded qubit subspace. Here we apply the same search for basis settings that optimize the gate noise in order to minimize qubits errors, as a figure of merit we use the error probability of Eq. (8). For easy comparison, the figures summarizing the different schemes considered and the resulting gate noise are also put together in Appendix C. The resulting error probabilities are then compared with the error probabilities for the DBSL in Sec. V A, while universality through the implementation of a non-Clifford gate in the various schemes is discussed in Sec. V B.

\section{A. Bilayer square lattice}

The two-dimensional BSL can be generated in the timefrequency domain using a single optical parametric oscillator [12] or solely in the time domain using four squeezing sources [16] as summarized in Fig. 6(a). We emphasize that the timeonly encoding of the BSL in [16] is not necessarily more favorable than the frequency-time encoding in [12]; one may even argue that the frequency-time encoding has a better scaling performance. Here we simply present the time-only encoded version of the setup, since it is comparable to that of the QRL and the MBSL, but it is important to note that the analysis presented in this work holds also for the timefrequency encoded BSL.

The setup in Fig. 6(a) produces a self-inverse and bipartite $\mathcal{H}$-graph state, which under phase rotations is transformed into a cluster state. An efficient universal computation scheme is well described by Alexander et al. $[12,16]$ in the language of macronodes in which each macronode corresponds to the logic level marked in Fig. 6(a). The computation takes place at this level and the logic cluster state consists of square cluster states as presented in Fig. 6(b) with $\pm t= \pm \tanh (2 r) / \sqrt{2}$ edge weight and $i \varepsilon=i \operatorname{sech} 2 r$ self-loops. The measuring system comprises two joint measurements for each temporal mode $k$ : a joint measurement of the control modes $B$ and $C$ in basis $(-1)^{k} \theta_{c}$ to project the cluster state into wires as shown in Fig. 6(c) and a joint measurement of the wire modes $A$ and $D$ to implement gates on these wires. As for the DBSL, we find that $\theta_{c}=\pi / 4$ is near optimal. Measuring wire modes $A$ and $B$ of temporal mode $k$ in bases $\theta_{A k}$ and $\theta_{D k}$ implements the 
(a)

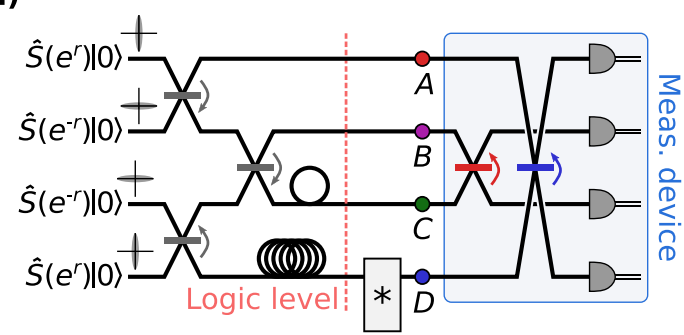

(b)

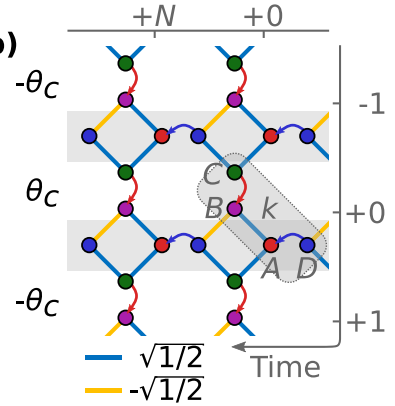

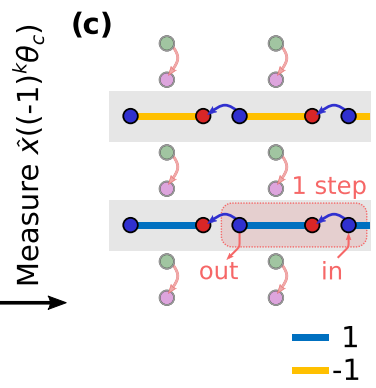

(d)

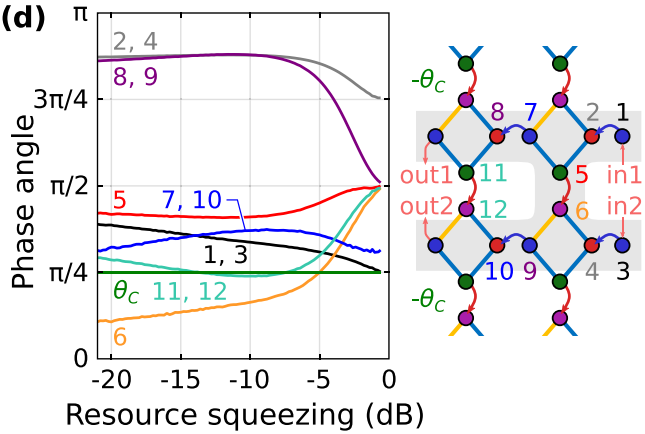

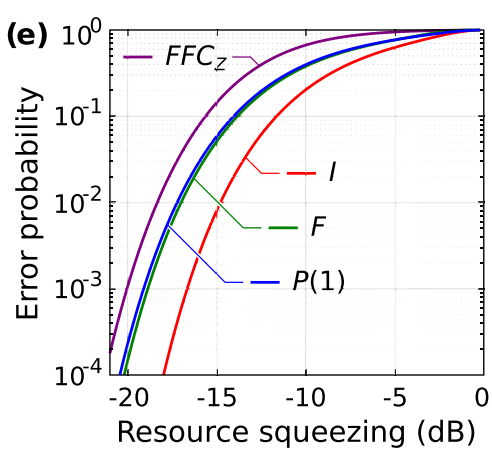

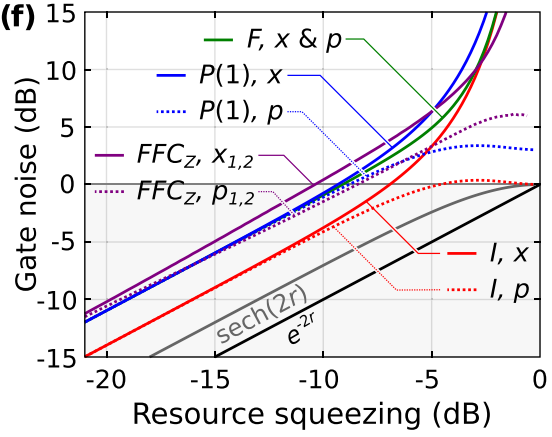

FIG. 6. Bilayer square lattice. (a) Experimental setup for generating the $\mathcal{H}$-graph state corresponding to the BSL cluster state [12,16]. Here the device marked by an asterisk is described in Fig. 2 for the DBSL and represents a switch for switching in and out states or a GKP quadrature correction circuit. The logic level in which the computation takes place is marked and the corresponding logic cluster state is shown in (b) with arrows representing the beam splitters of the measurement device, while in (c) the logic cluster state is projected into wires for computation. The edge weights shown here are in the limit of infinite squeezing and $\theta_{c}=\pi / 4$. (d) Basis setting implementing the $\left(\hat{F} \otimes \hat{F}^{-1}\right) \hat{C}_{Z}(1)$ gate for even temporal modes $k$ with a minimum error probability. For uneven $k,(\hat{F} \otimes \hat{F}) \hat{C}_{Z}(1)$ is implemented by changing the sign of the bases. The error probability in Eq. (8) of the single-mode $\hat{I}, \hat{F}$, and $\hat{P}(1)$ gates with $\theta_{c}=\pi / 4$ and the $\left(\hat{F} \otimes \hat{F}^{ \pm 1}\right) \hat{C}_{Z}(1)$ gate are presented in (e) with the corresponding gate noise shown in (f) Here $\hat{F} \hat{F} \hat{C}_{Z}$ is short for $\left(\hat{F} \otimes \hat{F}^{ \pm 1}\right) \hat{C}_{Z}(1)$. The experimental setup, logic cluster state and its projection into wires, and the resulting gate noise are shown together with the other schemes considered in Appendix $\mathrm{C}$ for easy comparison.

single-mode gate

$$
\hat{S}\left([-1]^{k+1} 2 t^{2}\right) \hat{R}\left(\frac{\theta_{+}}{2}\right) \hat{S}\left(\tan \frac{\theta_{-}}{2}\right) \hat{R}\left(\frac{\theta_{+}}{2}\right)
$$

from temporal mode $k$ to $k+N$ (one computation step) where $\theta_{ \pm}=\theta_{D k} \pm \theta_{A k}$. The resulting gate noise for one computation step is $\mathbf{N}\left(\hat{p}_{A k}, \hat{p}_{B k}, \hat{p}_{C k+1}, \hat{p}_{D k+N}\right)^{T}$, where

$$
\mathbf{N}=\left(\begin{array}{cccc}
\frac{1}{2 t^{2}} & \frac{1}{2 t} & -\frac{1}{2 t} & 0 \\
0 & -t & -t & 1
\end{array}\right) .
$$

Thus, the variance of the gate noise added to the output quadratures in each computation step is $N_{x} \varepsilon / 2$ and $N_{p} \varepsilon / 2$ for the $\hat{x}$ and $\hat{p}$ quadratures, respectively, where

$$
\begin{aligned}
& N_{x}=\frac{1}{\tanh ^{4} 2 r}+\frac{1}{\tanh ^{2} 2 r}, \\
& N_{p}=\tanh ^{2}(2 r)+1
\end{aligned}
$$

are quadrature noise factors $\sum_{j} N_{i j}^{2}$, introduced in Sec. II B 2, and we note that they are identical to the noise factors of the DBSL. The $\hat{I}$ gate is implemented in a single computation step by choosing

$$
\left(\begin{array}{l}
\theta_{+} \\
\theta_{-}
\end{array}\right)_{I}=\left(\begin{array}{c}
0 \\
(-1)^{k+1} 2 \arctan \left(\tanh ^{-2} 2 r\right)
\end{array}\right) .
$$

The $\hat{F}$ and $\hat{P}(1)$ gates are implemented in two computation steps from temporal mode $k$ to $k+2 N$. By choosing basis settings

$$
\left(\begin{array}{c}
\theta_{+1} \\
\theta_{-1} \\
\theta_{+2} \\
\theta_{-2}
\end{array}\right)_{F}=\left(\begin{array}{c}
\pi / 2 \\
\pi / 2 \\
0 \\
2 \arctan \left(\tanh ^{-4} 2 r\right)
\end{array}\right),
$$

$\hat{F}$ is implemented with equal gate noise variance of $\left(N_{x}+\right.$ $\left.N_{p}\right) \varepsilon / 2$ in $\hat{x}$ and $\hat{p}$ quadratures, while $\hat{P}(1)$ is realized with

$$
\left(\begin{array}{l}
\theta_{+1} \\
\theta_{-1} \\
\theta_{+2} \\
\theta_{-2}
\end{array}\right)_{P}=\left(\begin{array}{c}
\arctan 2 \\
-\arctan 2 \\
\pi / 2 \\
\pi / 2
\end{array}\right),
$$

resulting in gate noise variances of $2 N_{x} \varepsilon / 2$ and $2 N_{p} \varepsilon / 2$ in $\hat{x}$ and $\hat{p}$ quadratures, respectively. Here $\theta_{ \pm 1}=\theta_{D k} \pm \theta_{A k}$ and $\theta_{ \pm 2}=\theta_{D k+N} \pm \theta_{A k+N}$. Notice the similarity to the DBSL: The basis settings and gate noises are identical and the BSL and DBSL are expected to perform single-mode gates equally well.

Measuring control modes $B$ and $C$ of one temporal mode in different bases leads to coupling between the two neighboring wires and thus allow for the implementation of two-mode gates. In Ref. [12], the basis setting for implementing the $\hat{C}_{Z}(g)$ gate is given for the case of infinite squeezing. Here 
(a)

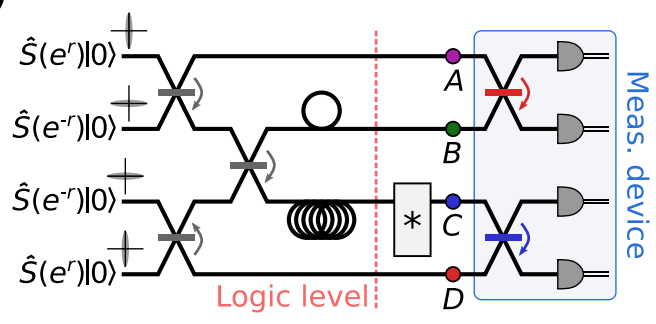

(b)

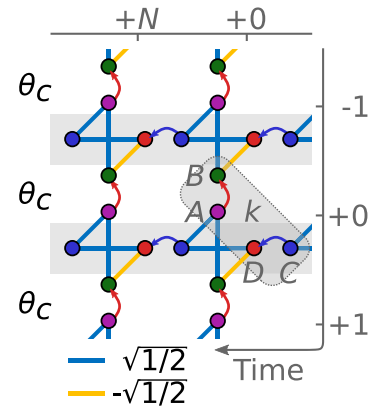

(c)

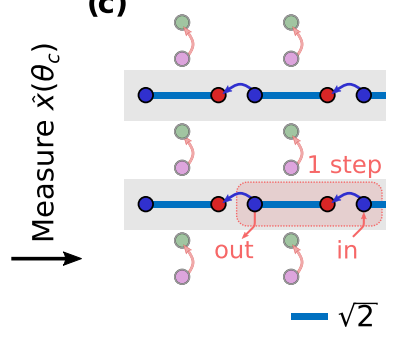

(d)

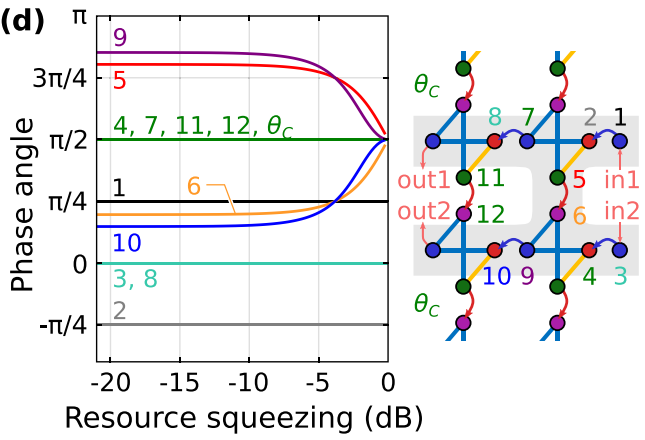

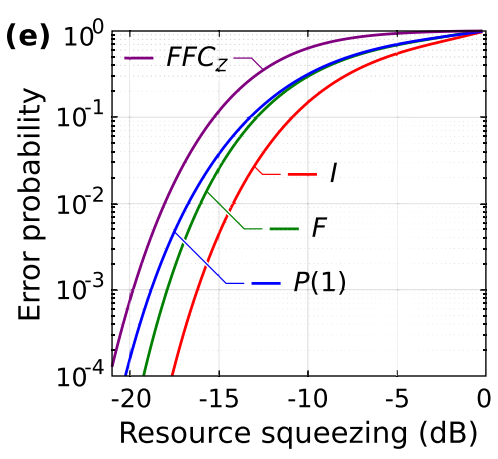

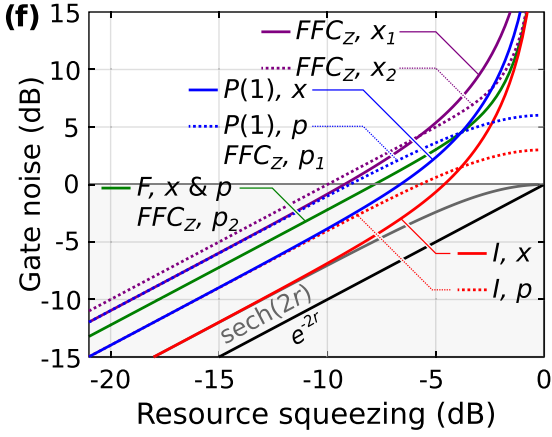

FIG. 7. Modified bilayer square lattice. (a) Experimental setup for generating the $\mathcal{H}$-graph state corresponding to the MBSL cluster state by Asavanant et al. [18]. Here the device marked by an asterisk is described in Fig. 2 for the DBSL and represents a switch for switching in and out states or a GKP quadrature corrections circuit. The logic level in which the computation takes place is marked and the corresponding logic cluster state is shown in (b) with arrows representing the beam splitters of the measurement device, while in (c) the logic cluster state is projected into wires for computation. The edge weights shown here are in the limit of infinite squeezing and $\theta_{c}=\pi / 2$. (d) Basis setting implementing the $(\hat{F} \otimes \hat{F}) \hat{C}_{Z}(1)$ gate with a minimum error probability. The error probability in Eq. (8) of the single-mode $\hat{I}, \hat{F}$, and $\hat{P}(1)$ gates with $\theta_{c}=\pi / 2$ and the $\left(\hat{F} \otimes \hat{F}^{ \pm 1}\right) \hat{C}_{Z}(1)$ gate are presented in (e) with the corresponding gate noise shown in (f). Here $\hat{F} \hat{F} \hat{C}_{Z}$ is short for $(\hat{F} \otimes \hat{F}) \hat{C}_{Z}(1)$. Note that each gate noise variance in the $\hat{p}$ quadratures of each mode for the $(\hat{F} \otimes \hat{F}) \hat{C}_{Z}(1)$ gate equals that of the $\hat{P}(1)$ and $\hat{F}$ gates. The experimental setup, logic cluster state and its projection into wires, and the resulting gate noise are shown together with the other schemes considered in Appendix C for easy comparison.

we extend this analysis by searching for the basis setting that minimizes the error probability of two encoded qubits after the $\hat{C}_{Z}(1)$ gate for the more relevant case of finite squeezing. To do so, we use the same technique as for the DBSL by minimizing the objective function in Eq. (13). Note that to compensate for finite squeezing distortion [as $\hat{S}\left( \pm \tanh ^{2} 2 r\right)$ in Eq. (14) for single-mode gates], two computation steps are required to implement $\hat{C}_{Z}(1)$. For all $\left(\hat{F}^{n} \otimes \hat{F}^{m}\right) \hat{C}_{Z}(1)$ gates with $n, m=0,1,2,3$ we find the lowest error probability for $n, m= \pm 1$ and we choose $(n, m)=\left(1,(-1)^{k+1}\right)$, where $k$ is the temporal mode index of the control modes coupling the two wires. The resulting basis settings implementing the $\left(\hat{F} \otimes \hat{F}^{-1}\right) \hat{C}_{Z}(1)$ gate are shown in Fig. 6(d) for even $k$, while for uneven $k$ the $(\hat{F} \otimes \hat{F}) \hat{C}_{Z}(1)$ gate is implemented with equal error probability by changing the sign of all bases in Fig. 6(d). If we allow for a variable $\theta_{c}$ in the objective function of Eq. (13), we find no improvement of the error probability, and we conclude there will be no gain of a variable $\theta_{c}$ when implementing the $\left(\hat{F} \otimes \hat{F}^{ \pm 1}\right) \hat{C}_{Z}(1)$ gate.

Note how the basis settings in Fig. 6(d), different from the DBSL in Fig. 4(a) and the MBSL later in Fig. 7(d), seem to depend on the resource squeezing in the full squeezing range shown. The reason for this is that there exist multiple solutions for basis settings that implement a desired $\hat{C}_{Z}(1)$ gate with a minimum error probability. The same is the case for the DBSL and MBSL; however, in Figs. 4(d) and 7(d) a more consistent solution set of basis settings as a function of resource squeezing is shown, while here for the BSL a slightly inconsistent solution set is shown. This effect often occurs when unnecessarily large degrees of freedom in the basis settings are used when minimizing the objective function in Eq. (13). However, this does not mean that the basis settings in Fig. 6(d) are not optimal, but are rather an example of the existence of multiple basis setting solutions and proper use of Eq. (13) to derive a suitable solution for a given experimental implementation.

The resulting error probabilities of Eq. (8) when correcting the quadratures after the $\hat{I}, \hat{F}, \hat{P}(1)$, and $\left(\hat{F} \otimes \hat{F}^{ \pm 1}\right) \hat{C}_{Z}(1)$ gates as described above are presented in Fig. 6(e). As expected, the two-mode $\hat{C}_{Z}(1)$ gate is seen to have the highest error probability since four successful quadrature corrections are necessary to avoid inducing an error on the encoded qubits. Finally, the gate noise variances are presented in Fig. 6(f), and here we clearly see behavior similar to that for the DBSL: For infinite squeezing, the $\hat{I}$ gate in one computation step has a gate noise variance of twice the effective variance squeezing $\operatorname{sech} 2 r$ (as $N_{x}, N_{p} \rightarrow 2$ when $r \rightarrow \infty$ ), while the $\hat{F}$ and $\hat{P}(1)$ gates implemented in two computation steps have gate noise variances four times that. In the other extreme of vanishing squeezing, the gate noise diverges in the $\hat{x}$ quadrature, thereby erasing all information of the encoded state as previously explained for the DBSL. This can also be seen from the corresponding Wigner function transformation in Appendix B. 


\section{B. Modified bilayer square lattice}

The experimental setup of the MBSL cluster state, recently generated by Asavanant et al. [18] and summarized in Fig. 7(a), is very similar to the setup of the all-time encoded BSL in Fig. 6(a), and we can therefore adopt the computation scheme for the BSL with only a few changes. The corresponding cluster state at the logic level is shown in Fig. 7(b), in which we see that the square clusters of the BSL have been replaced with "butterfly" clusters. As for the BSL, the edge weight and self-loops are $\pm t= \pm \tanh (2 r) / \sqrt{2}$ and $i \varepsilon=i \operatorname{sech} 2 r$, respectively. The spatial modes $C$ and $D$ of each temporal mode $k$ constitute wire modes, while $A$ and $B$ are control modes. In contrast to the square clusters in the BSL, the butterfly clusters already contain direct edges in the wires before potential phase rotation of the control modes. Thus we can directly delete the control modes by measuring them in the $\hat{x}$ basis, i.e., $\theta_{c}=0$, and implement the operations

$$
\hat{S}(t) \hat{R}\left(\frac{\theta_{+}}{2}\right) \hat{S}\left(\tan \frac{\theta_{-}}{2}\right) \hat{R}\left(\frac{\theta_{+}}{2}\right) \quad\left(\theta_{c}=0\right)
$$

in one computation step from temporal mode $k$ to $k+N$ with $\theta_{ \pm}=\theta_{C k} \pm \theta_{D k}$. The resulting gate noise is $\mathbf{N}\left(\hat{p}_{D k}, \hat{p}_{A k}, \hat{p}_{B k+1}, \hat{p}_{C k+N}\right)^{T}$, with

$$
\mathbf{N}=\left(\begin{array}{cccc}
-\frac{1}{t} & 0 & 0 & 0 \\
0 & 0 & 0 & 1
\end{array}\right) \quad\left(\theta_{c}=0\right)
$$

such that the gate noise variance is $N_{x} \varepsilon / 2$ and $N_{p} \varepsilon / 2$ in $\hat{x}$ and $\hat{p}$ quadratures, respectively, with quadrature noise factors of

$$
N_{x}=2 / \tanh ^{2} 2 r, \quad N_{p}=1 \quad\left(\theta_{c}=0\right) .
$$

Alternatively, we can measure the control modes in the $\hat{p}$ basis, i.e., $\theta_{c}=\pi / 2$, rearranging the edge weights of the butterfly cluster states to increase the edge weight between wire modes as shown in Fig. 7(c). In this case, the operation

$$
\hat{S}(2 t) \hat{R}\left(\frac{\theta_{+}}{2}\right) \hat{S}\left(\tan \frac{\theta_{-}}{2}\right) \hat{R}\left(\frac{\theta_{+}}{2}\right) \quad\left(\theta_{c}=\pi / 2\right)
$$

is implemented with the gate noise

$$
\mathbf{N}=\left(\begin{array}{cccc}
-\frac{1}{2 t} & -\frac{1}{2 t} & 0 & 0 \\
0 & 0 & -1 & 1
\end{array}\right) \quad\left(\theta_{c}=\pi / 2\right)
$$

such that

$$
N_{x}=1 / \tanh ^{2} 2 r, \quad N_{p}=2 \quad\left(\theta_{c}=\pi / 2\right) .
$$

Other values of $\theta_{c}$ are also possible, but in this case the implemented gate as well as gate noise is less trivial. However, in the later analysis of the $\hat{C}_{Z}(1)$ gate we do find that $\theta_{c}=\pi / 2$ is indeed optimal. Notice that, unlike the BSL with square cluster states, all control modes are measured in the same basis without an alternating sign for different temporal modes. This is because the wire modes are directly connected with equal edge weights for all temporal modes, or connected with three edges through two control modes, while for the square cluster states, wire modes have two connections, each through a single control mode, but with different sign on the edge weights depending on whether the control mode is in the next or previous temporal mode.
For $\theta_{c}=\pi / 2$, the basis setting

$$
\left(\begin{array}{c}
\theta_{+} \\
\theta_{-}
\end{array}\right)_{I}=\left(\begin{array}{c}
0 \\
2 \arctan \left[\tanh ^{-1}(2 r) / \sqrt{2}\right]
\end{array}\right)
$$

with $\theta_{ \pm}=\theta_{C k} \pm \theta_{D k}$ implements the $\hat{I}$ gate in one computation step from temporal mode $k$ to $k+N$ and gate noise variances $N_{x} \varepsilon / 2$ and $N_{p} \varepsilon / 2$ in $\hat{x}$ and $\hat{p}$ quadratures, respectively. The basis setting

$$
\left(\begin{array}{c}
\theta_{+1} \\
\theta_{-1} \\
\theta_{+2} \\
\theta_{-2}
\end{array}\right)_{F}=\left(\begin{array}{c}
\pi / 2 \\
\pi / 2 \\
0 \\
2 \arctan \left[\tanh ^{-2}(2 r) / 2\right]
\end{array}\right)
$$

implements the $\hat{F}$ gate in two computation steps with equal $\left(N_{x}+N_{p}\right) \varepsilon / 2$ gate noise variances in $\hat{x}$ and $\hat{p}$ quadratures, while

$$
\left(\begin{array}{l}
\theta_{+1} \\
\theta_{-1} \\
\theta_{+2} \\
\theta_{-2}
\end{array}\right)_{P}=\left(\begin{array}{c}
\arctan 2 \\
-\arctan 2 \\
\pi / 2 \\
\pi / 2
\end{array}\right)
$$

implements the $\hat{P}(1)$ gate in two computation steps with $2 N_{x} \varepsilon / 2$ and $2 N_{p} \varepsilon / 2$ gate noise variances in $\hat{x}$ and $\hat{p}$ quadratures, respectively. Here $\theta_{ \pm 1}=\theta_{C k} \pm \theta_{D k}$ and $\theta_{ \pm 2}=\theta_{C k+N} \pm$ $\theta_{D k+N}$ when implementing $\hat{F}$ and $\hat{P}(1)$ from temporal mode $k$ to $k+2 N$.

To couple pairs of wires for the implementation of a twomode gate, one measures the control modes $A$ and $B$ of one temporal mode $k$ in different bases by which a coupling between the wires in temporal modes $k-1$ and $k$ is induced. The $\hat{C}_{Z}(1)$ gate is again implemented in two computation steps, and similar as for the BSL and the DBSL, we search the basis setting that minimizes the objective function in Eq. (13) and thus the error probability in Eq. (8) of that particular gate. Again, we need to investigate all $\left(\hat{F}^{n} \otimes \hat{F}^{m}\right) \hat{C}_{Z}(1)$ gates for $n, m=0,1,2,3$ and find $n=m=1$ to be optimal. The resulting basis setting is shown in Fig. 7(d), where $\theta_{c}=\pi / 2$ is found to be optimal. Note that, unlike the DBSL and BSL, this basis setting is independent of the temporal mode index $k$, as the control basis does not have an alternating sign governed by $k$.

The resulting error probability of the single-mode $\hat{I}, \hat{F}$, and $\hat{P}(1)$ gates and the two-mode $(\hat{F} \otimes \hat{F}) \hat{C}_{Z}(1)$ gate, with the basis settings described above and in Fig. 7(d), is shown in Fig. 7(e). The single-mode gates are all seen to have a lower error probability than in computations with the DBSL and BSL cluster states. This is explained by the lower quadrature noise factors $N_{x}$ and $N_{p}$ due to the structure of the butterfly cluster states with initial edges between wire modes before projecting the logic cluster state into wires. As expected, due to the four quadrature corrections, the error probability of the $(\hat{F} \otimes \hat{F}) \hat{C}_{Z}(1)$ gate is largest. Gate noise variances are shown in Fig. 7(f). For single-mode gates, in general we see lower gate noise variance than for the DBSL and BSL, and in the large squeezing limit where $N_{x} \rightarrow 1$ for $r \rightarrow \infty$ and $\theta_{c}=\pi / 2$, while $N_{p}=2$, we see the gate noise variances in $\hat{x}$ quadratures of the $\hat{I}$ gate to equal the effective squeezing 
(a)

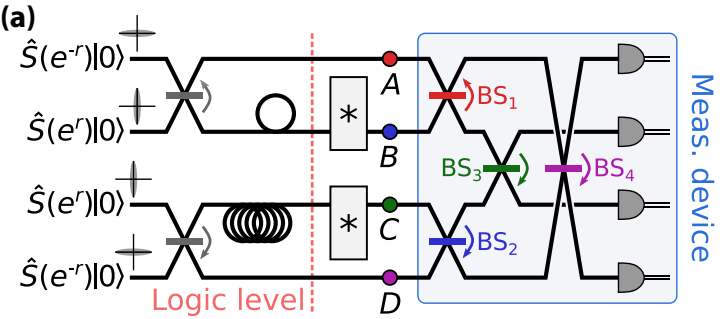

(c)

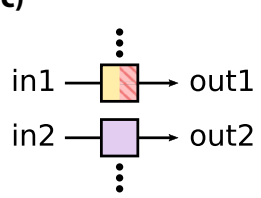

(b)

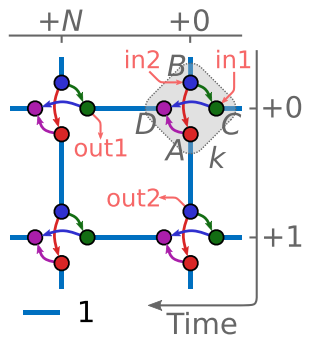

(d)

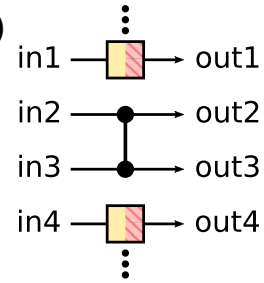

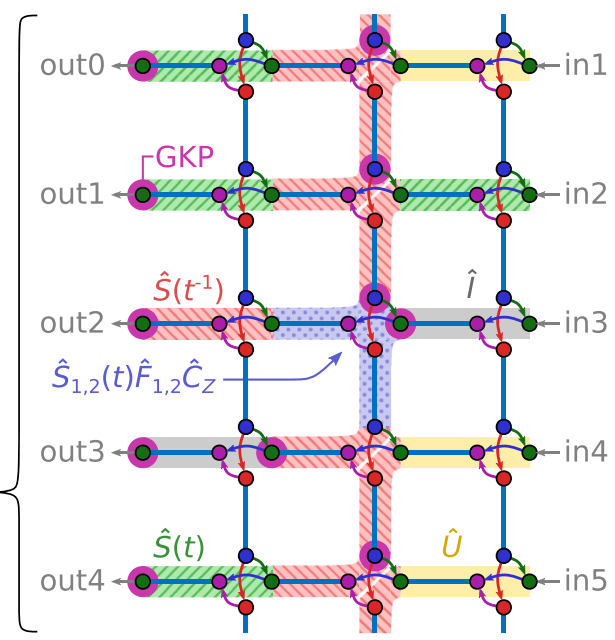

FIG. 8. Quad-rail lattice. (a) Experimental setup for generating the $\mathcal{H}$-graph state corresponding the QRL cluster state. Here the devices marked by an asterisk are described in Fig. 2 and represent optical switches switching in and out states or GKP quadrature correction circuits as in Eq. (7). The logic level in which the computation takes place is marked, with the corresponding logic cluster state shown in (b) with arrows representing the beam-splitter network of the measurement device. The edge weight shown is in the infinite squeezing limit, while for finite squeezing it is $t=\tanh 2 r$, while $i \varepsilon=i \operatorname{sech} 2 r$ self-loops are present on all nodes. Temporal mode indices are marked on the time axis, while a single computation step is marked with input modes in 1 and in 2 and output modes out 1 and out2. (c) Example of single-mode computation along the cluster state cylinder by restricting the bases with $\theta_{A k}=\theta_{D k}$ and $\theta_{B k}=\theta_{C k}$, thereby implementing $\hat{U}$ in Eq. (17) in each computation step. With two computation steps, the $\hat{S}(t)$ distortion in Eq. (17) due to finite squeezing can be compensated in the second step by implementing $\hat{S}\left(t^{-1}\right)$ or, more generally, any single-mode Gaussian gate can be implemented as $\hat{G}=\hat{U}_{1} \hat{U}_{2}$. Here input modes in spatial modes $B$ are ignored. After implementation of gates, the output modes marked with an outer circle are quadrature corrected. (d) Example of implementing the $(\hat{F} \otimes \hat{F}) \hat{C}_{Z}(1)$ gate between input modes in2 and in3. Since all gates are implemented on pairs of modes, first one computation step is required to guide the in 2 and in 3 modes to the $(\hat{F} \otimes \hat{F}) C Z(1)$ gate, while gates of the form $\hat{S}\left(t^{-1}\right) \hat{U}$ can be implemented on other computation modes. After the $(\hat{F} \otimes \hat{F}) C Z(1)$ gate, all computation modes are aligned to the same vertical position in the lattice using $\hat{I}$ gates [notice that $\hat{S}\left(t^{ \pm 1}\right) \hat{S}\left(t^{\mp 1}\right)=\hat{I}$ ]. To prevent accumulating gate noise, GKP quadrature correction is performed after every implemented gate on modes marked with an outer circle. The experimental setup and logic cluster state are shown together with the other schemes considered in Appendix $\mathrm{C}$ for easy comparison.

variance of $\operatorname{sech} 2 r$. For vanishing squeezing, the gate noise variance diverges in the $\hat{x}$ quadrature, erasing all information of the encoded state as is also the case for computing with the BSL and DBSL (also eluded by the Wigner function transformation in Appendix B). Notice that, unlike the DBSL and BSL, the gate noise of the $(\hat{F} \otimes \hat{F}) \hat{C}_{Z}(1)$ gate is not symmetric in the quadratures of the two modes.

\section{Quad-rail lattice}

In Ref. [14] it was proposed to generate a cluster state with a quad-rail lattice structure in the frequency domain from a single optical parametric oscillator, while in Ref. [13] it was suggested to construct a time domain version of the QRL clusters state. With temporal encoding, the generated state has a cylindrical topology reminiscent of the DBSL, BSL, and MBSL, allowing for computation along the cylinder with information encoded on the circumference of the cylinder. The scheme for generating the temporally encoded QRL state is summarized in Fig. 8(a). Since the QRL is self-inverse and bipartite, this QRL $\mathcal{H}$-graph state has a corresponding QRL cluster state (under phase rotations), which we consider in the following.

An efficient computation scheme on the QRL cluster state is presented in [19] in the language of macronodes. It corresponds to the logic level marked in Fig. 8(a), which is followed by a measurement device consisting of a beamsplitter network of four beam splitters $\left(\mathrm{BS}_{1-4}\right)$ and four homodyne detections. The cluster state at the logic level is shown in Fig. 8(b). With the logic level at a beam-splitter depth of only one, the edge weight of $t=\tanh 2 r$ is larger than in the DBSL, BSL, and MBSL, while the self-loops are equal $i \varepsilon=i \operatorname{sech} 2 r$. The logic cluster state consists of two-mode entangled states as in the generalized teleportation circuit in Sec. II B, and no projection of the cluster state into wires before computation is necessary. This, together with the larger edge weight, reduces the gate noise and thus makes computation on the QRL more efficient. On the other hand, the increased complexity of the measurement device (a joint measurements of four modes) makes the computation scheme presented here more tricky and may seem less intuitive.

One computation step is marked in Fig. 8(b). It implements a two-mode operation from input modes $C k$ (in1) and $B k$ (in2) to the output modes $C k+N$ (out1) and $B k+1$ (out2). In the following we will refer to the mode in computation from in1 (in2) to out1 (out2) as computation mode 1 (2). It is possible to decouple the two computation modes 1 and 2 by restricting the basis settings to $\theta_{A k}=\theta_{D k}$ and $\theta_{B k}=\theta_{C k}$. In the same way as in Eq. (9), this effectively cancels the beam splitters $\mathrm{BS}_{3}$ and $\mathrm{BS}_{4}$, since equal phase shifts commute with the beam splitter. Then single-mode gates can be implemented using $\mathrm{BS}_{1}$ and $\mathrm{BS}_{2}$ in the same way as for the generalized 
teleportation in Sec. II B, but the same gate will be applied to both computation modes 1 and 2 due to the basis restriction. That is, $\hat{U} \otimes \hat{U}$ will be implemented, where

$$
\hat{U}=\hat{S}(\tanh 2 r) \hat{R}\left(\frac{\theta_{+}}{2}\right) \hat{S}\left(\tan \frac{\theta_{-}}{2}\right) \hat{R}\left(\frac{\theta_{+}}{2}\right),
$$

with $\theta_{ \pm}=\theta_{C k} \pm \theta_{D k}$. Similarly, using the basis permutation rules in [19], restricting to $\theta_{D k}=\theta_{B k}$ and $\theta_{A k}=\theta_{C k}$ implements $\hat{U} \otimes \hat{U}$ on modes from in 1 and in 2 to out 2 and out 1 , respectively. As a result, when implementing $\hat{U} \otimes \hat{U}$, the modes in computation may travel straight across each other on the cluster state lattice or they may do a $90^{\circ}$ change in their computation direction on the lattice depending on the basis restriction. For implementation of single-mode gates, we will mainly focus on the former case in which one mode (computation mode 1) travels in the direction of the cluster state cylinder, while the other mode (computation mode 2), traveling around the cylinder, is ignored, as illustrated in Fig. 8(c). Regardless of the basis restriction, the gate noise of one computation step is $\mathbf{N}\left(\hat{p}_{A k}, \hat{p}_{D k}, \hat{p}_{B k+1}, \hat{p}_{C k+N}\right)^{T}$, with

$$
\mathbf{N}=\left(\begin{array}{cccc}
-\frac{1}{\tanh 2 r} & 0 & 0 & 0 \\
0 & -\frac{1}{\tanh 2 r} & 0 & 0 \\
0 & 0 & 1 & 0 \\
0 & 0 & 0 & 1
\end{array}\right)
$$

leading to equal quadrature noise factors in the two computation modes of

$$
N_{x}=\frac{1}{\tanh ^{2} 2 r}, \quad N_{p}=1
$$

in $\hat{x}$ and $\hat{p}$ quadratures, respectively.

As for the generalized teleportation circuit, the singlemode $\hat{I}$ gate is implemented in a single computation step with basis setting

$$
\left(\begin{array}{l}
\theta_{+} \\
\theta_{-}
\end{array}\right)_{I}=\left(\begin{array}{c}
0 \\
2 \arctan \left(\tanh ^{-1} 2 r\right)
\end{array}\right)
$$

with gate noise variances $N_{x} \varepsilon / 2$ and $N_{p} \varepsilon / 2$ in $\hat{x}$ and $\hat{p}$ quadratures, respectively. The $\hat{F}$ and $\hat{P}(1)$ gates are implemented in two computation steps: With the basis setting

$$
\left(\begin{array}{l}
\theta_{+1} \\
\theta_{-1} \\
\theta_{+2} \\
\theta_{-2}
\end{array}\right)_{F}=\left(\begin{array}{c}
\pi / 2 \\
\pi / 2 \\
0 \\
2 \arctan \left(\tanh ^{-2} 2 r\right)
\end{array}\right) \text {, }
$$

$\hat{F}$ is implemented with equal gate noise variances in $\hat{x}$ and $\hat{p}$ of $\left(N_{x}+N_{p}\right) \varepsilon / 2$, while

$$
\left(\begin{array}{l}
\theta_{+1} \\
\theta_{-1} \\
\theta_{+2} \\
\theta_{-2}
\end{array}\right)_{P}=\left(\begin{array}{c}
\arctan 2 \\
-\arctan 2 \\
\pi / 2 \\
\pi / 2
\end{array}\right)
$$

implements $\hat{P}(1)$ with gate noise variances of $2 N_{x} \varepsilon / 2$ and $2 N_{p} \varepsilon / 2$ in $\hat{x}$ and $\hat{p}$, respectively. Here, for the mode in computation traveling straight along the cylinder, $\theta_{ \pm 1}=\theta_{C k} \pm \theta_{D k}$ and $\theta_{ \pm 2}=\theta_{C k+N} \pm \theta_{D k+N}$, while $\left(\theta_{B k}, \theta_{A k}\right)=\left(\theta_{C k}, \theta_{D k}\right)$ and $\left(\theta_{B k+N}, \theta_{A k+N}\right)=\left(\theta_{C k+N}, \theta_{D k+N}\right)$.

To implement the $\hat{C}_{Z}(1)$ gate, we have investigated $\left(\hat{F}^{n} \otimes\right.$ $\left.\hat{F}^{m}\right) \hat{C}_{Z}(1)$ for $n, m=0,1,2,3$ and find that $n=m=1$ leads to the lowest error probability in Eq. (8) of the GKP-encoded qubits. With the basis setting

$$
\left(\begin{array}{c}
\theta_{A k} \\
\theta_{B k} \\
\theta_{C k} \\
\theta_{D k}
\end{array}\right)_{C_{Z}}=\left(\begin{array}{c}
\pi / 2-\arctan \frac{1}{2} \\
0 \\
\pi / 2+\arctan \frac{1}{2} \\
0
\end{array}\right),
$$

$[\hat{S}(t) \otimes \hat{S}(t)](\hat{F} \otimes \hat{F}) \hat{C}_{Z}(1)$ is implemented in a single computation step, where the two modes in computation goes from in 1 and in 2 to out 2 and out1, respectively (i.e., they do not cross, but each mode is redirected $\left.90^{\circ}\right)$. Here $[\hat{S}(t) \otimes \hat{S}(t)]$ is the distortion due to finite squeezing and is compensated for in each computation mode in a second computation step with basis setting

$$
\left(\begin{array}{l}
\theta_{+} \\
\theta_{-}
\end{array}\right)_{S\left(t^{-1}\right)}=\left(\begin{array}{c}
0 \\
2 \arctan \left(\tanh ^{-2} 2 r\right)
\end{array}\right) .
$$

As a result, $(\hat{F} \otimes \hat{F}) \hat{C}_{Z}(1)$ is implemented in two computation steps with equal gate noise variance in all four quadratures of $\left(N_{x}+N_{p}\right) \varepsilon / 2$ as for the $\hat{F}$ gate. As gates on the QRL are in general performed on pairs of modes in computation and require two computation steps (with the exception of the $\hat{I}$ gate), implementing $(\hat{F} \otimes \hat{F}) \hat{C}_{Z}(1)$ among other computation modes may be tricky. However, an example of a possible implementation is shown in Fig. 8(d).

The gate noise variance for each of the implemented gates in $\left\{\hat{I}, \hat{F}, \hat{P}(1),(\hat{F} \otimes \hat{F}) \hat{C}_{Z}(1)\right\}$ is shown in Fig. 9(a) as a function of the initial squeezing of the $\hat{p}$-quadrature variance in the resource state $e^{-2 r}$. Notice that in the high squeezing limit, the gate noise of the $\hat{I}$ gate is equal to the effective variance squeezing of the cluster state modes $\operatorname{sech} 2 r$, which is better than seen for the other computation schemes presented in this work and is due to the large edge weight in the logic cluster state with no projection of the cluster state necessary before computation. The $\hat{F}$ and $\hat{P}(1)$ gates, implemented in two computation steps, naturally have double gate noise compared to the $\hat{I}$ gate and so does the $(\hat{F} \otimes \hat{F}) \hat{C}_{Z}(1)$ gate, unlike the $\hat{C}_{Z}(1)$ gates implemented on the DBSL, BSL, and MBSL. This improvement for the $(\hat{F} \otimes \hat{F}) \hat{C}_{Z}(1)$ gate happens because no extra control modes are included when coupling two computation modes. In the limit of vanishing resource squeezing, the gate noise variance of each computation diverges in the $\hat{x}$ quadrature, erasing all information of the encoded state as for the generalized teleportation circuit in Eq. (5).

To prevent gate noise accumulating on the GKP-encoded qubits, quadrature corrections as described in Sec. II C should be performed on modes in computation after each implemented gate. Here, with two computation modes in each computation step, two quadrature correction devices are necessary: one in each spatial mode $B$ and $C$ as marked in Fig. 8(a). After quadrature correcting modes as shown in the examples in Figs. 8(c) and 8(d), the error probabilities of Eq. (8) are shown in Fig. 9(b) for each of the four gates $\hat{I}, \hat{F}, \hat{P}(1)$, and $(\hat{F} \otimes \hat{F}) \hat{C}_{Z}(1)$. As expected, with four 

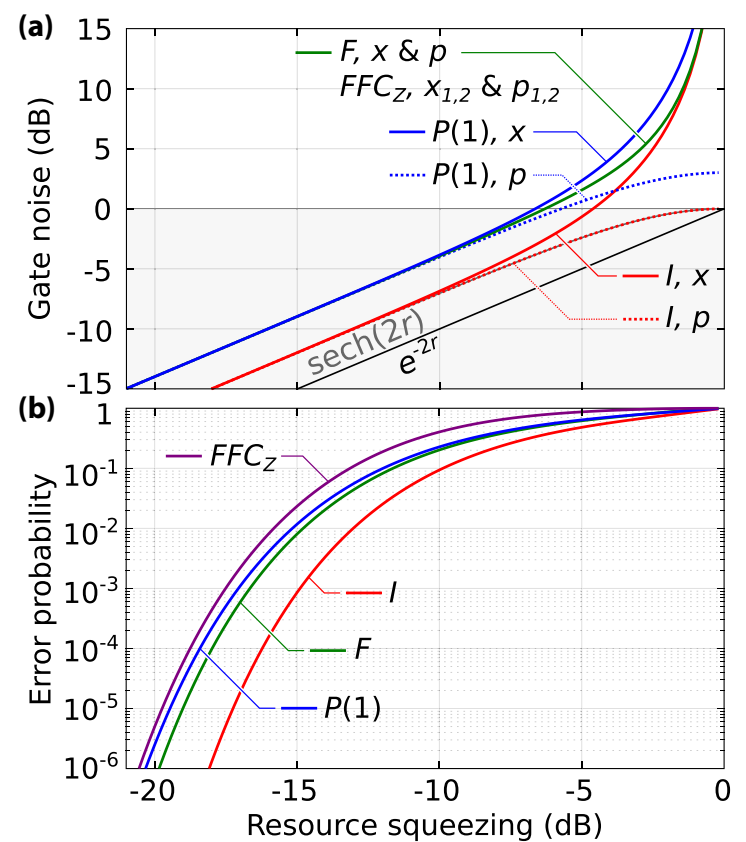

FIG. 9. (a) Gate noise variance of the $\hat{I}, \hat{F}, \hat{P}(1)$, and $(\hat{F} \otimes \hat{F})$ $\hat{C}_{Z}(1)$ gates (in short $\hat{F} \hat{F} \hat{C}_{Z}$ ) on the QRL cluster state as functions of input resource squeezing in Fig. 8(a). Here $e^{-2 r}$ and $\operatorname{sech} 2 r$ mark the resource and effective squeezing variance. Note that the gate noise variance in each of the four quadratures when implementing $\hat{F} \hat{F} \hat{C}_{Z}$ is equal to the gate noise variance when implementing the $\hat{F}$ gate. The gate noise here is shown together with the other schemes considered in Appendix $\mathrm{C}$ for easy comparison. (b) Resulting error probabilities of Eq. (8) after quadrature corrections.

successful quadrature corrections required to avoid qubit error, the $(\hat{F} \otimes \hat{F}) \hat{C}_{Z}(1)$ gate has the highest error probability. In Sec. V A it is compared with the DBSL, BSL, and MBSL.

\section{DISCUSSION}

Below, in Sec. VA we compare the cluster states and computation schemes presented and discussed in Secs. III and $\mathrm{IV}$, while the figures summarizing the different computation schemes and resulting gate noise are shown in Appendix C. In Sec. V B we then comment on computation universality with these cluster states.

\section{A. Cluster state comparison}

For all four cluster states considered in Secs. III and IV, the implemented two-mode $\hat{C}_{Z}(1)$ gates lead to the highest error probability of the GKP-encoded qubits among the gates of the set $\left\{\hat{I}, \hat{F}, \hat{P}(1), \hat{C}_{Z}(1)\right\}$. An indicative measure of the performance of a particular cluster state for quantum computing is thus the error probability associated with the implementation of the $\hat{C}_{Z}$ (1) gate. In Fig. 10(a) these are plotted for the DBSL, BSL, MBSL, and QRL. Here the error probability of $\hat{C}_{Z}(1)$ implemented on a canonically generated square lattice (SL) cluster state in [22] is plotted for comparison.

As discussed in Sec. II C, the error probability in Eq. (8) is fueled by the gate noise, the noise of the GKP qubits, and the noise introduced in quadrature error correction. Gate noise is governed by the amount of squeezing of the cluster state,
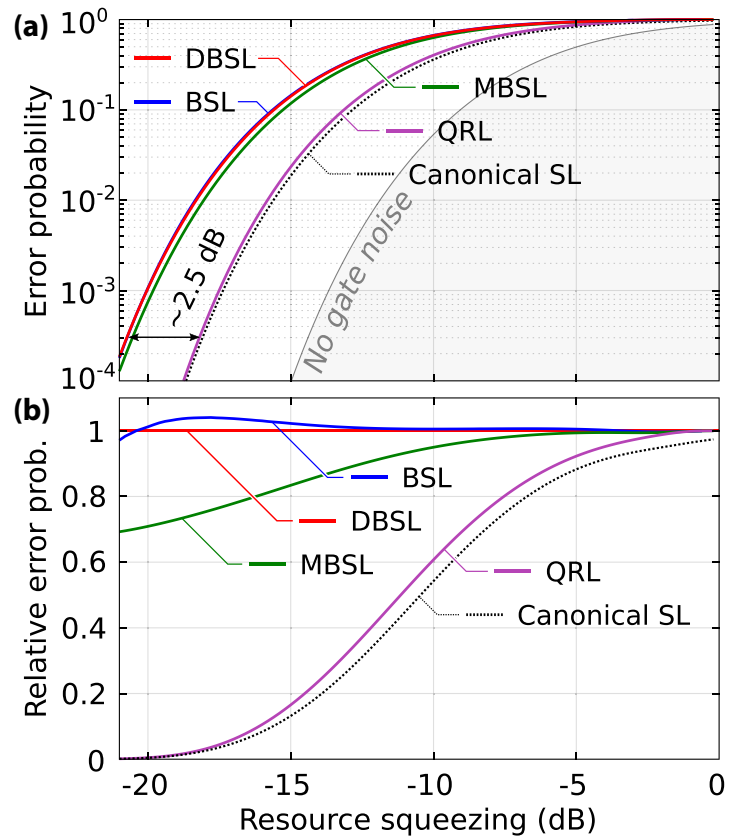

FIG. 10. (a) Error probabilities for $\hat{C}_{Z}(1)$ gates implemented on the DBSL in Sec. III, the BSL, the MBSL and QRL in Sec. IV, and the canonically generated square lattice cluster state in [22]. Depending on the cluster state, the implemented $\hat{C}_{Z}(1)$ gates have a Fourier gate by-product on each mode. Note that the error probabilities for the DBSL and BSL are overlapping. The gray area marks the error probability in the case of zero gate noise, where qubit errors are caused only by the available squeezing in the GKP encoding. (b) Error probabilities in (a) relative to that of the DBSL.

while the noise of the qubits and correction is produced by the finite squeezing of the peaks in the GKP state. Here, as described in Sec. III B, we have assumed the peak variances of both quadratures in the GKP states to equal the squeezing resource variance of $e^{-2 r} / 2$. To see how much the finite squeezing in the GKP encoding and correction contributes to the error probability, the $\hat{C}_{Z}(1)$ error probability in the case of zero gate noise [corresponding to setting $\boldsymbol{\sigma}^{2}=\mathbf{0}$ in Eq. (6)] is also plotted in Fig. 10(a). No matter what computation scheme is considered with the GKP encoding used here, we will not be able to perform better than the case of zero gate noise, as the noise contributions from the GKP encoding and quadrature correction are unavoidable.

The DBSL, BSL, and MBSL are seen to have similar performances, while the QRL is superior and almost matches the performance of the canonically generated SL cluster state. Approximately $2.5 \mathrm{~dB}$ of additional squeezing is necessary in the squeezing resources for the DBSL and BSL to match the performance of the QRL. This performance advantage of the QRL is due to the larger cluster state edge weight in the logic level and that the cluster state needs no projection by measurement of control modes, which adds additional noise to the state in computation. It is worth considering whether similar computation schemes can be developed for the DBSL, BSL, and MBSL, possibly by placing the logic levels closer to the squeezing sources in the setups after the first beam splitters (leading to temporally delocalized macronodes in the macronode language). 
To quantify further the performance difference of the DBSL, BSL, and MBSL, the $\hat{C}_{Z}(1)$ error probabilities are plotted in Fig. 10(b) relative to the $\hat{C}_{Z}(1)$ error probability of the DBSL. Here the BSL and DBSL are seen to have very similar performances in the range of resource squeezing investigated. The MBSL performs better with an error probability down to $70 \%$ of the error probability in the DBSL at $21 \mathrm{~dB}$ resource squeezing, while the relative error probability is approximately $83 \%$ using the currently achievable squeezing of $15 \mathrm{~dB}$ [37]. However, in practice, one also has to account for experimental imperfections and setup complexity when deciding which setup to use: The generation scheme of the DBSL is technically simpler than that of MBSL as it requires only two squeezing sources and three interference points contra four squeezing sources and five interference points.

At first sight, with only two squeezing sources, the DBSL seems to require fewer resources than the BSL and MBSL. However, in the DBSL cluster state, only every second temporal mode holds control modes, while for the BSL and MBSL every single temporal mode includes both control and wire modes. As a result, the DBSL only contains half as many modes for computation in the same number of temporal modes. Doubling the long delay $N \tau$ in the generation setup in Fig. 2(a) doubles the cylindrical cluster state circumference and compensates for only having wire modes in every second temporal mode. However, by doubling the circumference, the time needed to implement gates doubles as well. As a result, there is a cost of using only two squeezing sources in the DBSL, which unfolds as fewer computation modes or longer computation time, but not as additional computation noise.

Finally, we compare the architecture of the computation schemes on the cluster states considered. The DBSL, BSL, and MBSL all use the same principles of measuring control modes to control coupling between wires with modes in computation. Turning on and off coupling between wires makes it intuitive to implement multimode gates decomposed into single- and two-mode gates, while on the QRL one has to take care of the surrounding modes when implementing two-mode gates as for the $\hat{C}_{Z}(1)$ gate in Fig. 8(d). However, the control-mode-based architectures only allow coupling between neighboring wires, whereas the QRL is more "flexible" as introduced in [19]. As an example, consider an arbitrary SWAP gate $\hat{X}_{i j}$ swapping the modes in computation on wires $i$ and $j$. On the DBSL, a SWAP gate (with an unimportant Fourier gate applied to the two output modes) can be performed between two neighboring wires in two computation steps from temporal mode $(k-2, k)$ to $(k+2 N, k+2 N-2)$ with the basis setting

$$
\left(\begin{array}{c}
\theta_{A k-2} \\
\theta_{B k-2} \\
\theta_{A k} \\
\theta_{B k} \\
\theta_{A k+N-2} \\
\theta_{B k+N-2} \\
\theta_{A k+N-1} \\
\theta_{B k+N-1} \\
\theta_{A k+N} \\
\theta_{B k+N}
\end{array}\right)_{X}\left(\begin{array}{c}
\pi / 4 \\
-\pi / 4 \\
\pi / 4 \\
-\pi / 4 \\
\pi / 2 \\
0 \\
\pi / 2 \\
0 \\
\pi / 2 \\
0
\end{array}\right)
$$
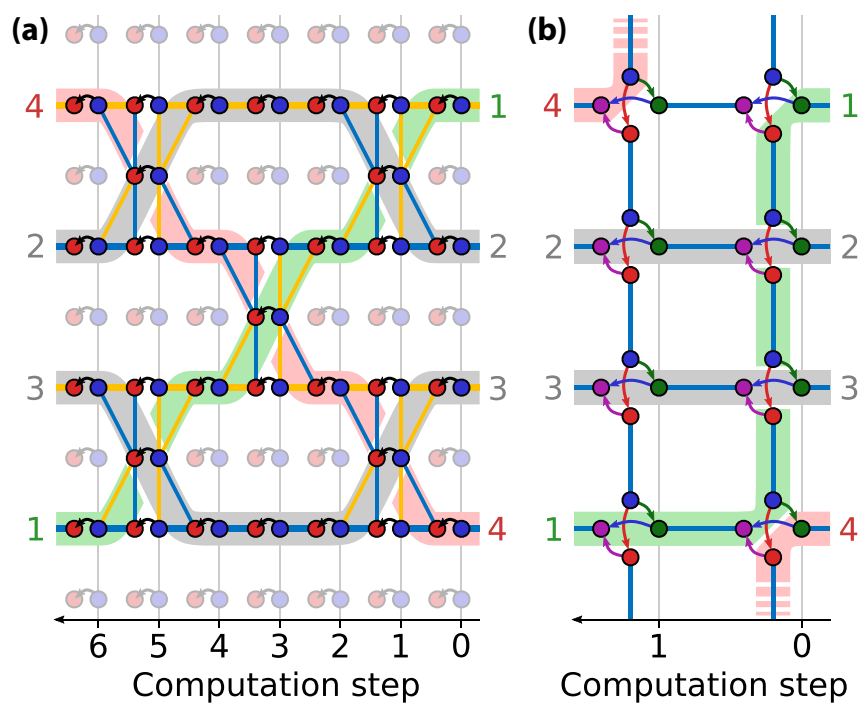

FIG. 11. Implementation of a SWAP gate between computation modes 1 and $4, \hat{X}_{14}$, separated by computation modes 2 and 3, on (a) the DBSL and (b) the QRL. Since only coupling between neighboring wires is possible on the DBSL in (a), a circuit depth of 3 is required, corresponding to six horizontal computation steps necessary along the cylindrical cluster state. The same goes for the BSL and the MBSL. On the QRL in (b), the same SWAP gate can be implemented in a single horizontal computation step using crossing identity gates $\hat{I}$. Here computation mode 1 crosses computation modes 2 and 3, while computation mode 4 is led all the way around the cluster state cylinder to appear in the next horizontal computation step, crossing other computation modes on its way.

independent of the amount of resource squeezing and with gate noise variances $N_{x} \varepsilon / 2$ and $N_{p} \varepsilon / 2$ in $\hat{x}$ and $\hat{p}$ quadratures, respectively, where $N_{x}=\tanh ^{-4}(2 r)+3 \tanh ^{-2} 2 r$ and $N_{p}=$ $\tanh ^{2}(2 r)+3$. Thus, to swap two modes on wires separated by $n$ wires in between, $n+1$ SWAP gates are required on each mode leading to $2(n+1)$ required computation steps. On the QRL, on the other hand, using vertically traveling modes in Fig. 8, two modes can be swapped in only a single horizontal computation step independent on the initial distance between the modes on the cluster state lattice. This is illustrated in Fig. 11 for $\hat{X}_{14}$ with a mode distance of 2 . As a result, depending on the interconnectivity required in the quantum algorithm to be implemented, computation times can be shorter on the QRL than on the DBSL, BSL, and MBSL.

Finally, we want to comment on the performance of the QRL compared to the canonically generated SL cluster state. It is clear that the QRL performs almost as well as the SL, while the SL is much more challenging to generate since it requires on-line squeezing to perform canonical $\hat{C}_{Z}(g)$ operations and the total squeezing cost is in general larger [3,38]. However, for a fair comparison, it should be mentioned that the $\hat{C}_{Z}(1)$ implemented in [22] on the SL was not optimized. It was implemented with four computation steps, where each of the $\hat{x}$ and $\hat{p}$ quadrature corrections in the GKP scheme was performed in two different computation steps, both leading to more noise on the GKP-encoded qubits. The $\hat{C}_{Z}(1)$ error probability on the SL may be improved by optimizing the required basis settings to implement the $\hat{C}_{Z}(1)$ gate in fewer 
computation steps and performing GKP quadrature corrections of both the $\hat{x}$ and $\hat{p}$ quadratures on the last cluster state mode as for the computation schemes considered in this work.

\section{B. Towards universality and fault tolerance}

The four different computation schemes in Secs. III and IV involve only Gaussian measurements (in the form of homodyne detection) on Gaussian cluster states. In this pure Gaussian realm, one is only able to perform universal Gaussian computation [3], which with Gaussian input modes may be simulated classically $[39,40]$. To achieve universal quantum computation, non-Gaussian operations or resources are required [41]. There exist different proposals on how to achieve a universal gate set, which we summarize and discuss in the following. Non-Gaussianity of states and operations has been obtained in numerous systems [42], including some recent results on optical non-Gaussian state preparation [43] and non-Gaussian transformations on cluster states [44].

In many $\mathrm{CV}$ quantum computing architecture proposals, the Gaussian gate set is complemented with the non-Gaussian cubic phase gate $\hat{K}(\chi)=e^{i \chi \hat{x}^{3} / 3}[3,27]$ to achieve universal quantum computation on the bosonic modes [41]. Such a non-Gaussian gate can be implemented, for example, by redirecting specific modes of the cluster to a photon counter, thereby realizing a measurement-induced non-Gaussian gate transformation [3]. Moreover, in [16] it was shown how $\hat{K}(\chi)$ may be implemented on the BSL using an ancillary cubic phase state $|\chi\rangle=\int d s e^{i \chi s^{3} / 3}|s\rangle_{x}$ as a non-Gaussian resource switched into the logic level of the computation scheme as an input state. Such a cubic phase state may be prepared using photon counting [27]. Given the similarities between computations on the BSL, the DBSL, and the MBSL, it is straightforward to adopt this method of implementing $\hat{K}(\chi)$ by inputting $|\chi\rangle$ in these computation schemes. A similar approach may also be viable on the QRL.

Using GKP states with symmetric quadrature noise, one can expect a bad performance of the cubic phase gate due to the applied phase by $\hat{K}(\chi)$ on the finitely squeezed GKP peaks being a cubic function of $\hat{x}[27,45]$. A more efficient approach to quantum universality is to consider a gate set that is only universal in the encoded logic space rather than in the full infinite-dimensional Hilbert space. This requires an ample supply of qubit magic states such as the Hadamard eigenstates $\left|H_{L}\right\rangle=\cos \pi / 8\left|0_{L}\right\rangle+\sin \pi / 8\left|1_{L}\right\rangle$. By injecting these states into the computation wires as input states using an optical switch, the non-Clifford $\pi / 8$ gate can be executed with only Gaussian transformations of the bosonic modes [27,46]. Such magic GKP states may be prepared similarly to the GKPencoded input states or directly distilled using GKP $\left|0_{L}\right\rangle$ states [47]. In conclusion, the inherent non-Gaussianity of the GKP states is sufficient to achieve universal quantum computation in the GKP-encoded qubit subspace using solely Gaussian transformations. Moreover, adding magic state distillation to the scheme may not increase the experimental requirements significantly since the squeezing needed for the distillation is expected to be lower than the squeezing already required to reach fault-tolerant Clifford computation [22,47].
Finally, for fault-tolerant computation, the qubit errorcorrection scheme, concatenated with the GKP errorcorrection scheme, should be considered when estimating the required squeezing. With the quadrature corrections of states in computation after each implemented gate, gate noise and finite squeezing in the approximate GKP-encoded qubit states are translated to qubit errors. For fault-tolerant computation, these qubit errors are corrected with an appropriate qubit error-correction scheme, where a logic qubit is encoded in multiple GKP qubits. Here it is not appropriate just to choose a qubit error-correction scheme with a large qubit error threshold, as considerations on how to practically implement the scheme are also of critical importance. For this reason, here we will not estimate a squeezing threshold for fault tolerance. As an example, the seven-qubit Steane code with an $10^{-3}$ error threshold requires two-mode gates between arbitrary modes in computation [28], while the computation schemes discussed in Secs. III and IV only implement two-mode gates between neighboring computation modes. Thus, to implement the seven-qubit Steane code a number of SWAP gates are required for each syndrome measurement, each leading to an increase in the combined qubit error probability before qubit error correction. The QRL may have an advantage when considering the implementation of a qubit error-correction scheme owing to its flexibility as previously discussed and illustrated for a SWAP gate in Fig. 11. Future work includes considerations on the practical implementation of qubit error correction on suitable and realizable cluster states.

\section{CONCLUSION}

In summary, we have reviewed the principles of $\mathrm{CV}$ measurement-based QC based on generalized teleportation, we have proposed an efficient computation scheme for the DBSL cluster state that was experimentally generated in [17], and we have carefully analyzed and compared quantum computation based on that state with the BSL, QRL, and MBSL cluster states.

Through a careful study of the added gate noise for the different cluster states, we find that the DBSL, the BSL, and the MBSL exhibit similar performances. We also find that the QRL is superior in terms of performance and flexibility, allowing implementation of quantum circuits in a minimum number of time steps. Finally, we have reviewed proposals for implementation of a universal gate set, either on the bosonic modes or just in the GKP-encoded qubit subspace, and concluded that universal qubit computation is possible in all four cluster states considered, given the availability of GKP states.

To optimize the performance of the various computation schemes, we introduced a tool to find the basis setting implementing a desired gate with minimum GKP-encoded qubit errors. We believe that this technique for finding the optimal basis settings will be important for future developments and optimizations of new types of gates and algorithms. It should however be noted that the technique of optimizing the basis setting might not be the only strategy for minimizing the error probability: We have only considered GKP qubit encodings on a square grid in phase space, which is appropriate for symmetric noise addition among conjugate quadratures. However, since the computation schemes considered in 
general add noise asymmetrically in the quadratures, it may be beneficial to encode the qubits in a rectangular lattice. Since different gates have different gate noise asymmetry in $\hat{x}$ and $\hat{p}$ quadratures, the optimal lattice ratio depends on which gates dominate the circuit to be implemented: As an example, the Fourier gate $\hat{F}$ in general adds symmetric gate noise, in which case a square lattice is optimal, while the gate noise asymmetry of the identity gate $\hat{I}$ depends on the resource squeezing. One can argue that with the $\hat{C}_{Z}$ gate being the noisiest gate, the GKP lattice ratio should be optimized to minimize qubit error for this gate. In this case, for the DBSL, BSL, and MBSL, the optimal ratio again depends on the resource squeezing, while for the superior QRL with symmetric gate noise, the square lattice seems optimal. However, one further complication is that when performing the $\hat{C}_{Z}$ gate or the $\hat{P}$ gate, not only is gate noise added, but also noise from the state in computation is added due to the addition of quadratures in these gates. Thus, the optimal lattice ratio depends as well on the noise performance of the states in computation and the determination of a general optimal lattice ratio for a given application is outside the scope of this work. Finally, one has to keep in mind that changing the lattice ratio also alters the logic operators in the GKP encoding. As an example, with a rectangular lattice the logic Hadamard gate becomes a combination of the Fourier and squeezing gates.

Throughout this article, we have assumed all cluster states to be pure, while in practice the cluster state will have some degree of mixedness in the form of excess noise in the antisqueezed quadratures. However, it was shown in Ref. [48] that excess noise in the antisqueezed quadrature does not affect the performance of the computation, and thus our purity assumption in this article is well justified. It is however worth mentioning that in practice it is still favorable to produce highly pure squeezed states as large excess noise will decrease the amount of squeezing due to inevitable phase instabilities of the experimental setup.

In this article we have not studied the actual implementation of qubit error correction. Thus, as an outlook, it would be interesting to study how a qubit error-correction algorithm is most efficiently implemented such that the squeezing threshold for fault-tolerant quantum computation is minimized. An interesting solution could be topological QC, for which the resulting squeezing threshold is within the already experimentally demonstrated range [49,50]. In topological QC, the qubits are encoded in a two-dimensional plane while the actual computation takes place in a third dimension, thus rendering the need for the construction of 3D cluster states. Proposals do exist for the generation of 3D cluster states [51,52], and the next interesting step is thus to analyze the performance of these states using the techniques developed in this article.

\section{ACKNOWLEDGMENTS}

We acknowledge useful discussion with Rafael N. Alexander and Peter van Loock. The work was supported by the Danish National Research Foundation through the Center for Macroscopic Quantum States (bigQ) through Grant No. DNRF0142.

\section{APPENDIX A: CALCULATION OF QUADRATURE TRANSFORMATIONS}

In this Appendix we present an example of the quadrature transformation of the single-mode computation step on the DBSL that leads to the expressions (10) and (12) in Sec. III. The modes involved are shown on the graph in Fig. 12(a) with the corresponding circuit in Fig. 12(b). We will use the mode numbering labeled in Fig. 12(a).

The approximate cluster state (ancillary modes $2-7$ ) consists of vacuum states squeezed by $\sqrt{\varepsilon}$ and connected by $\hat{C}_{Z}(g)$ operations of weights that are described by the adjacency matrix

$$
\mathbf{A}=\left(\begin{array}{ccccccc}
0 & 0 & 0 & 0 & 0 & 0 & 0 \\
0 & 0 & t & t & t & -t & 0 \\
0 & t & 0 & 0 & 0 & 0 & -t \\
0 & t & 0 & 0 & 0 & 0 & -t \\
0 & t & 0 & 0 & 0 & 0 & t \\
0 & t & 0 & 0 & 0 & 0 & -t \\
0 & 0 & -t & -t & t & -t & 0
\end{array}\right) .
$$

Thus, in the Heisenberg picture, we consider the generation of the cluster state as a quadrature transformation described by the symplectic matrix

$$
\mathbf{S}_{C Z}=\left(\begin{array}{ll}
\mathbf{I} & \mathbf{0} \\
\mathbf{A} & \mathbf{I}
\end{array}\right)
$$

on the input and initially squeezed ancillary modes, where $\mathbf{I}$ and $\mathbf{0}$ are the $7 \times 7$ identity and zero matrix, respectively (note that the quadratures of the input mode 1 are left unchanged by $\mathbf{S}_{C Z}$ ). The input mode 1 is then connected to the cluster state by a beam splitter [the beam splitter of the measurement device in Fig. 2(a)], leading to the quadrature transformation

$$
\mathbf{S}_{B S}=\left(\begin{array}{ll}
\mathbf{B} & \mathbf{0} \\
\mathbf{0} & \mathbf{B}
\end{array}\right),
$$

where

$$
\mathbf{B}=\left(\begin{array}{ccccccc}
1 / \sqrt{2} & -1 / \sqrt{2} & 0 & 0 & 0 & 0 & 0 \\
1 / \sqrt{2} & 1 / \sqrt{2} & 0 & 0 & 0 & 0 & 0 \\
0 & 0 & 1 & 0 & 0 & 0 & 0 \\
0 & 0 & 0 & 1 & 0 & 0 & 0 \\
0 & 0 & 0 & 0 & 1 & 0 & 0 \\
0 & 0 & 0 & 0 & 0 & 1 & 0 \\
0 & 0 & 0 & 0 & 0 & 0 & 1
\end{array}\right) .
$$

Here we have ignored beam-splitter operations on the control modes 3-6, as these operations can be directly compensated for by adding or subtracting the measurement outcomes of the homodyne detectors as shown in Eq. (9). Finally, each mode $j$, except the output mode 7 , is measured in basis $\hat{x}\left(\theta_{j}\right)$. This is represented first by a phase rotation $\hat{R}\left(\theta_{j}\right)$, followed by a homodyne measurement of $\hat{x}_{j}$. Thus, before the $\hat{x}$ measurements, the quadratures are transformed as

$$
\mathbf{S}_{R}=\left(\begin{array}{cc}
\mathbf{c} & \mathbf{s} \\
-\mathbf{s} & \mathbf{c}
\end{array}\right),
$$

where $\mathbf{c}$ and $\mathbf{s}$ are matrices with $\left(\cos \theta_{1}, \ldots, \cos \theta_{7}\right)$ and $\left(\sin \theta_{1}, \ldots, \sin \theta_{7}\right)$ in the diagonal, respectively, and zero elsewhere. For implementing single-mode gates, the control mode measurement bases are set to $\left(\theta_{3}, \theta_{4}, \theta_{5}, \theta_{6}\right)=$ $(-1)^{i} \theta_{c}(1,1,-1,-1)$, where $\theta_{c}=\pi / 4$ for simplicity, and 
(a)

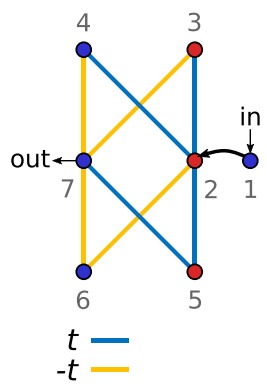

(b)

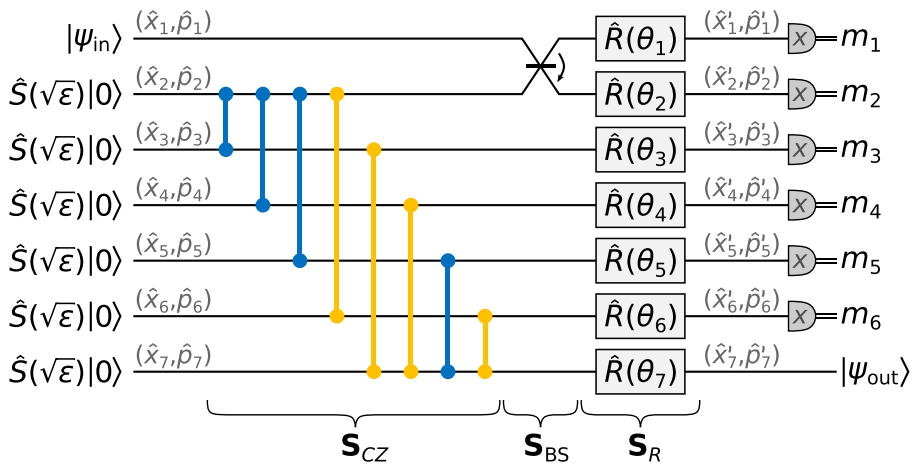

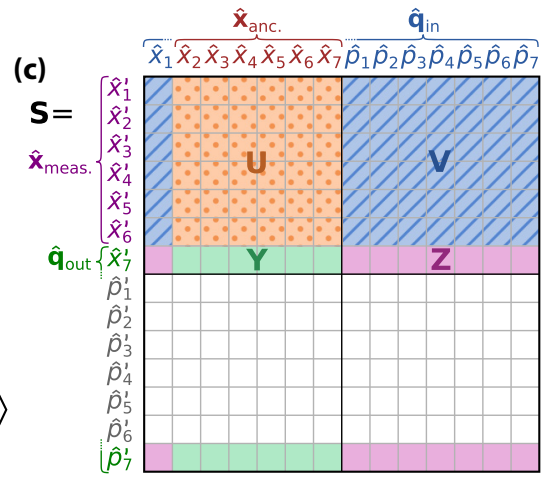

FIG. 12. (a) Graphical notation of a single-mode computation step on the DBSL. For an input in temporal mode $k$, the mode numbering translates as $(1,2,3,4,5,6,7)=(B k, A k, A k-1, B k+N-1, A k+1, B k+N+1, B k+N)$, where $A$ and $B$ are spatial modes in Fig. 2(a). (b) Corresponding circuit where the blue (dark) and yellow (bright) two-mode gates represent $\hat{C}_{Z}(g)$ gates of weights $t$ and $-t$, respectively. (c) Outline of the symplectic matrix $\mathbf{S}=\mathbf{S}_{R} \mathbf{S}_{B S} \mathbf{S}_{C Z}$ representing the quadrature transformation in (b).

the output mode cannot be phase rotated, $\theta_{7}=0$. Here $i$ is the wire number as shown in Fig. 2(b). The total quadrature transformation of the input and squeezed cluster state modes just before measurements is then

$$
\hat{\mathbf{q}}^{\prime}=\mathbf{S}_{R} \mathbf{S}_{B S} \mathbf{S}_{C Z} \hat{\mathbf{q}}=\mathbf{S} \hat{\mathbf{q}}
$$

where $\quad \hat{\mathbf{q}}=\left(\hat{x}_{1}, \ldots, \hat{x}_{7}, \hat{p}_{1}, \ldots, \hat{p}_{7}\right)^{T} \quad$ and $\quad \hat{\mathbf{q}}^{\prime}=$ $\left(\hat{x}_{1}^{\prime}, \ldots, \hat{x}_{7}^{\prime}, \hat{p}_{1}^{\prime}, \ldots, \hat{p}_{7}^{\prime}\right)^{T}$ are vectors of quadrature operators before and after the transformation as marked in Fig. 12(b). It should be noted that for the cluster state prepared as an $\mathcal{H}$ graph with off-line squeezing only, the effective amount of squeezing of the cluster state modes is $\varepsilon=\operatorname{sech} 2 r$, where $r$ is the squeezing parameter of the initially prepared off-line squeezed state with variance $e^{-2 r}$ [3].

Next we solve for the antisqueezed $\hat{x}$ quadratures of the cluster state modes $\hat{\mathbf{x}}_{\text {anc }}=\left(\hat{x}_{2}, \ldots, \hat{x}_{7}\right)^{T}$ as a function of the measured $\hat{x}$ quadratures $\hat{\mathbf{x}}_{\text {meas }}=\left(\hat{x}_{1}^{\prime}, \ldots, \hat{x}_{6}^{\prime}\right)^{T}$,

$$
\begin{aligned}
\hat{\mathbf{x}}_{\text {meas }} & =\mathbf{U} \hat{\mathbf{x}}_{\text {anc }}+\mathbf{V} \hat{\mathbf{q}}_{\text {in }} \\
& \text { 业 } \\
\hat{\mathbf{x}}_{\text {anc }} & =\mathbf{U}^{-1} \hat{\mathbf{x}}_{\text {meas }}-\mathbf{U}^{-1} \mathbf{V} \hat{\mathbf{q}}_{\text {in }},
\end{aligned}
$$

where $\hat{\mathbf{q}}_{\text {in }}=\left(\hat{x}_{1}, \hat{p}_{1}, \ldots, \hat{p}_{7}\right)^{T}$, while $\mathbf{U}$ and $\mathbf{V}$ are the parts of $\mathbf{S}$ that transform $\hat{\mathbf{x}}_{\text {anc }}$ and $\hat{\mathbf{q}}_{\text {in }}$ to $\hat{\mathbf{x}}_{\text {meas }}$ as shown in Fig. 12(c). Finally, we substitute the $\hat{x}$ quadratures of the cluster state with the quadratures of output mode $7, \hat{\mathbf{q}}_{\text {out }}=\left(\hat{x}_{7}^{\prime}, \hat{p}_{7}^{\prime}\right)$ :

$$
\begin{aligned}
\hat{\mathbf{q}}_{\text {out }} & =\mathbf{Y} \hat{\mathbf{x}}_{\text {anc }}+\mathbf{Z} \hat{\mathbf{q}}_{\text {in }} \\
& =\mathbf{Y}\left(\mathbf{U}^{-1} \hat{\mathbf{x}}_{\text {meas }}-\mathbf{U}^{-1} \mathbf{V} \hat{\mathbf{q}}_{\text {in }}\right)+\mathbf{Z} \hat{\mathbf{q}}_{\text {in }} \\
& =\left(\mathbf{Z}-\mathbf{Y} \mathbf{U}^{-1} \mathbf{V}\right) \hat{\mathbf{q}}_{\text {in }}+\mathbf{Y} \mathbf{U}^{-1} \hat{\mathbf{x}}_{\text {meas }}
\end{aligned}
$$

With $\hat{\mathbf{x}}_{\text {meas }} \rightarrow\left(m_{1}, \ldots, m_{6}\right)$ when measuring, $\mathbf{Y} \mathbf{U}^{-1} \hat{\mathbf{x}}_{\text {meas }}$ corresponds to the by-product displacement, while $\mathbf{M} \equiv \mathbf{Z}-$ $\mathbf{Y} \mathbf{U}^{-1} \mathbf{V}$ of size $2 \times 8$ corresponds to the combined gate symplectic matrix $\mathbf{G}$ and gate noise matrix $\mathbf{N}$ in Eq. (2) as $\mathbf{M}=(\mathbf{G} \mathbf{N})$. Extracting $\mathbf{G}$ as the first two columns of $\mathbf{M}$ transforming $\left(\hat{x}_{1}, \hat{p}_{1}\right)$ to $\left(\hat{x}_{7}^{\prime}, \hat{p}_{7}^{\prime}\right)$, we get

$$
\mathbf{G}=\frac{1}{\sin \theta_{-}}\left(\begin{array}{cc}
\frac{1}{t^{\prime}} \cos \theta_{+}+\frac{1}{t^{\prime}} \cos \theta_{-} & \frac{1}{t^{\prime}} \sin \theta_{+} \\
-t^{\prime} \sin \theta_{+} & t^{\prime} \cos \theta_{+}-t^{\prime} \cos \theta_{-}
\end{array}\right),
$$

which is the symplectic matrix corresponding to the operation in Eq. (10) where $t^{\prime}=(-1)^{i} 4 t^{2}$ and $\theta_{ \pm}=\theta_{1} \pm \theta_{2}$. Further, $\mathbf{N}$ is associated with the remaining six columns of $\mathbf{M}$,

$$
\mathbf{N}=\left(\begin{array}{cccccc}
-\frac{1}{4 t^{2}} & \frac{1}{4 t} & \frac{1}{4 t} & -\frac{1}{4 t} & \frac{1}{4 t} & 0 \\
0 & t & t & t & -t & 1
\end{array}\right)
$$

which leads to the quadrature noise factors coined in Eq. (12) when $t=\tanh (2 r) / 2$.

The procedure shown here for calculating the gate symplectic matrix $\mathbf{G}$ and gate noise matrix $\mathbf{N}$ is not limited to the single-mode one computation step on the DBSL but represents a general procedure that can be used to analyze the noise of all gates in this work (irrespective of the cluster state) as done in Secs. III and IV: If $\mathbf{S}_{C Z}$ represents the construction of any cluster state and $\mathbf{S}_{R} \mathbf{S}_{B S}$ represents any Gaussian measurement, we can determine the resulting linear quadrature transformation corresponding to an arbitrary Gaussian operation on a single-mode or multimode input state. For each case, we need to keep track of the following quadratures: $\hat{\mathbf{x}}_{\text {anc }}$ including antisqueezed $\hat{x}$ quadratures of the cluster state, $\hat{\mathbf{q}}_{\text {in }}$ including the input mode quadratures and squeezed $\hat{p}$ quadratures of the cluster state leading to gate noise, $\hat{\mathbf{x}}_{\text {meas }}$ including the transformed $\hat{x}$ quadratures to be measured, and $\hat{\mathbf{q}}_{\text {out }}$ including the output mode quadratures of nonmeasured modes.

\section{APPENDIX B: WIGNER FUNCTION TRANSFORMATIONS}

In this Appendix we discuss the single-mode computation step in the DBSL, BSL, and MBSL in the Wigner function representation. Here, for simplicity, the basis setting for implementing the $\hat{I}$ gate is chosen, while to shorten the notation, we have postselected on measurement outcomes equal zero. As described in Sec. II B, nonzero measurement outcomes 
lead to an unimportant displacement in phase space. For the QRL, the two-mode cluster state corresponds to the one considered for the generalized teleportation in Sec. II B, and so the Wigner function transformation is similar to that presented in Eq. (5).
For a single-mode $\hat{I}$ gate performed on the DBSL in one computation step, described in Secs. III A and III B, the transformation of the Wigner function can be calculated in the same way as we did for the generalized teleportation in Sec. II B, resulting in

$$
\begin{aligned}
W_{\text {out }}(x, p)= & \mathcal{N} G_{1 / \varepsilon}(x) \int d \eta_{4} G_{\varepsilon}\left(\eta_{4}\right) G_{4 t^{2} / \varepsilon}\left(p-\eta_{4}\right) \int d \eta_{3} G_{\varepsilon / 4 t^{2}}\left(\eta_{3}\right) \\
& \times G_{1 / 4 t^{2} \varepsilon}\left(x-\eta_{3}\right) \int d \eta_{2} G_{4 t^{2} \varepsilon}\left(\eta_{2}\right) G_{16 t^{4} / \varepsilon}\left(p-\eta_{2}-\eta_{4}\right) \int d \eta_{1} G_{\varepsilon / 16 t^{4}}\left(\eta_{1}\right) W_{\text {in }}\left(\begin{array}{c}
x-\eta_{1}-\eta_{3} \\
p-\eta_{2}-\eta_{4}
\end{array}\right),
\end{aligned}
$$

where $\mathcal{N}$ is a normalization factor and $G_{\delta}$ is a normalized Gaussian function of $\delta / 2$ variance. The transformation includes two convolutions in each quadrature and corresponding envelopes in the conjugate quadrature due to the Fourier relation between quadratures. Comparing with Eq. (A1) and referring to the mode numbering in Fig. 12(a), in the $\hat{x}$ quadrature the first convolution with $G_{\varepsilon / 16 t^{4}}$ corresponds to noise from the finitely squeezed mode 2, while the third convolution with $G_{\varepsilon / 4 t^{2}}$ corresponds to noise of control modes 3-6. In the $\hat{p}$ quadrature, the second convolution with $G_{4 t^{2} \varepsilon}$ corresponds to noise from the control modes 3-6, while the last convolution with $G_{\varepsilon}$ corresponds to the finite squeezing noise of the output mode 7. In the limit of infinite squeezing $r \rightarrow \infty$ (assuming $t \neq 0$ ), the convolution functions become $\delta$ functions since $\varepsilon=\operatorname{sech} 2 r \rightarrow 0$, while their corresponding envelopes in the orthogonal quadratures become infinitely broad, and so $W_{\text {out }}(x, p) \rightarrow W_{\text {in }}(x, p)$. In the limit of $t=0$ where we expect no information to pass from the input mode 1 to the output mode 7 , the first three convolutions lead to the Wigner function of an infinitely squeezed state in $\hat{p}$, erasing all information of the input state, while the last convolution with $G_{\varepsilon}$ in $\hat{p}$ quadrature ensures that the output Wigner function equals the initial squeezed Wigner function of mode $7, W_{\text {out }}(x, p)=G_{1 / \varepsilon}(x) G_{\varepsilon}(p)$, which equals vacuum for no squeezing as $\varepsilon=\operatorname{sech} 2 r=1$ when $r=0$.

On the BSL, the single-mode $\hat{I}$ gate performed in one computation step transforms the Wigner function as

$$
\begin{aligned}
W_{\text {out }}(x, p)= & \mathcal{N} G_{1 / \varepsilon}(x) \int d \eta_{4} G_{\varepsilon}\left(\eta_{4}\right) G_{2 t^{2} / \varepsilon}\left(p-\eta_{4}\right) \int d \eta_{3} G_{\varepsilon /\left(2 t^{2}\right)}\left(\eta_{3}\right) \\
& \times G_{1 / 2 t^{2} \varepsilon}\left(x-\eta_{3}\right) \int d \eta_{2} G_{2 t^{2} \varepsilon}\left(\eta_{2}\right) G_{4 t^{2} / \varepsilon}\left(p-\eta_{2}-\eta_{4}\right) \int d \eta_{1} G_{\varepsilon / 4 t^{4}}\left(\eta_{1}\right) W_{\text {in }}\left(\begin{array}{c}
x-\eta_{1}-\eta_{3} \\
p-\eta_{2}-\eta_{4}
\end{array}\right) .
\end{aligned}
$$

Similar to the DBSL, comparing with $\mathbf{N}$ in Eq. (15), the convolutions with $G_{\varepsilon / 4 t^{4}}$ and $G_{\varepsilon / 2 t^{2}}$ in the $\hat{x}$ quadrature correspond, respectively, to noise added from the first wire mode $A k$ and the two control modes $B k+1$ and $C k+N$ in a square cluster of the BSL in Figs. 6(a) and 6(b). In the $\hat{p}$ quadrature, the convolution with $G_{2 t^{2} \varepsilon}$ corresponds to noise from the control modes $B k+1$ and $C k+N$, while the convolution with $G_{\varepsilon}$ corresponds to noise from the output mode $D k+N$. Again, in the limit of infinite squeezing, $W_{\text {out }}(x, p) \rightarrow W_{\text {in }}(x, p)$, while for $t=0$ the output Wigner function becomes $W_{\text {out }}(x, p)=G_{1 / \varepsilon}(x) G_{\varepsilon}(p)$, as expected.

For the MBSL, the Wigner function transformation of the $\hat{I}$ gate in one computation step with the control basis $\theta_{c}=\pi / 2$ is

$$
\begin{aligned}
W_{\text {out }}(x, p)= & \mathcal{N} G_{1 / \varepsilon}(x) G_{2 \varepsilon+4 t^{2} / \varepsilon}(p) \int d \eta_{3} G_{\varepsilon / 4 t^{2}}\left(\eta_{3}\right) G_{1 / \varepsilon}\left(x-2 \eta_{3}\right) \int d \eta_{2} G_{2 \varepsilon}\left(\eta_{2}\right) \\
& \times G_{4 t^{2} / \varepsilon}\left(p-\eta_{2}\right) \int d \eta_{1} G_{\varepsilon / 4 t^{2}}\left(\eta_{1}\right) W_{\text {in }}\left(\begin{array}{c}
x-\eta_{1}-\eta_{3} \\
p-\eta_{2}
\end{array}\right) .
\end{aligned}
$$

Due to the direct edges along the computation wires of the butterfly cluster states in Fig. 7, the Wigner function transformation becomes less intuitive. Here the envelope $G_{2 \varepsilon+4 t^{2} / \varepsilon}(p)$ corresponds to an envelope of $G_{4 t^{2} / \varepsilon}(p)$ convoluted with $G_{2 \varepsilon}$. Comparing with Eq. (16), the first and third convolutions in the $\hat{x}$ quadrature, both with $G_{\varepsilon / 4 t^{2}}$, correspond to noise added from wire mode $D k$ and control mode $A k$. The second convolution with $G_{2 \varepsilon}$ in the $\hat{p}$ quadrature corresponds to noise from both control mode $B k+1$ and the output mode $C k+N$. In the infinite squeezing limit, $W_{\text {out }}(x, p) \rightarrow W_{\text {in }}(x, p)$. For $t=0$ we get $W_{\text {out }}(x, p)=G_{1 / \varepsilon}(x) G_{2 \varepsilon}(p) G_{2 \varepsilon}(p)=G_{1 / \varepsilon}(x) G_{\varepsilon}(p)$.

If instead of the identity gate $\hat{I}$ an arbitrary singlemode Gaussian gate of one computation step is implemented with symplectic matrix $\mathbf{G}$, the resulting Wigner function transformation corresponds to that presented above, but with the arguments of $W_{\text {in }}$ transformed by $\mathbf{G}^{-1}$ as shown in Eq. (5) for the generalized teleportation. For single-mode gates implemented in two computation steps, the output Wigner function of the first step becomes the input Wigner function of the second step, leading to addition of the gate noise variance since a convolution of two Gaussian functions is a Gaussian function with the combined variance, i.e., additive Gaussian gate noise. For multimode gates, more modes are involved, leading to more convolutions in the expression of the output Wigner function, and the Wigner function representation becomes tedious. However, the principle is the same as for single-mode gates: The gate noise leads to convolutions with Gaussian functions of variance equal the gate noise variance. 

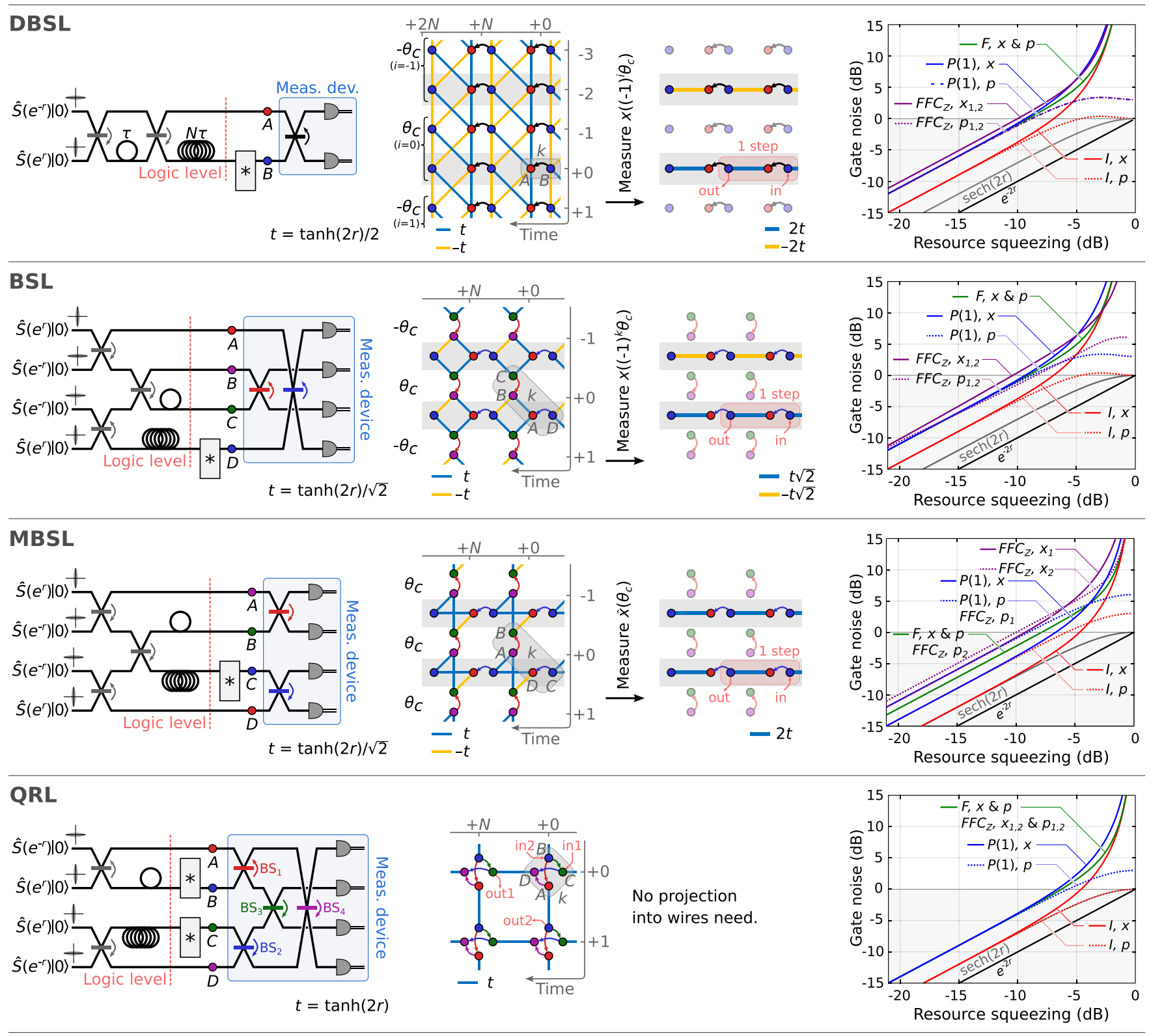

FIG. 13. Setup, cluster state in the logic level, and resulting gate noise of each DBSL, BSL, MBSL, and QRL cluster state studied in Secs. III and IV. For the DBSL, BSL, and MBSL, the projection into wires is shown as well, while this is not required for computation on the QRL.

\section{APPENDIX C: CLUSTER STATE COMPARISON CHEAT SHEET}

In Secs. III and IV the computation schemes on the different cluster states are analyzed separately in order to facilitate easy lookup of a specific cluster state. The different schemes are discussed and compared in Sec. V, while in this Appendix the schemes are arranged in Fig. 13 for easy comparison.
[1] R. Raussendorf and H. J. Briegel, A One-Way Quantum Computer, Phys. Rev. Lett. 86, 5188 (2001).

[2] N. C. Menicucci, P. van Loock, M. Gu, C. Weedbrook, T. C. Ralph, and M. A. Nielsen, Universal Quantum Computation with Continuous-Variable Cluster States, Phys. Rev. Lett. 97, 110501 (2006).

[3] M. Gu, C. Weedbrook, N. C. Menicucci, T. C. Ralph, and P. van Loock, Quantum computing with continuous-variable clusters, Phys. Rev. A 79, 062318 (2009).
[4] Y. Miwa, J. I. Yoshikawa, P. van Loock, and A. Furusawa, Demonstration of a universal one-way quantum quadratic phase gate, Phys. Rev. A 80, 050303(R) (2009).

[5] Y. Wang, X. Su, H. Shen, A. Tan, C. Xie, and K. Peng, Toward demonstrating controlled-X operation based on continuous variable four-partite cluster state and quantum teleporters, Phys. Rev. A 81, 022311 (2010).

[6] R. Ukai, N. Iwata, Y. Shimokawa, S. C. Armstrong, A. Politi, J. I. Yoshikawa, P. van Loock, and A. Furusawa, Demonstration 
of Unconditional One-Way Quantum Computations for Continuous Variables, Phys. Rev. Lett. 106, 240504 (2011).

[7] R. Ukai, S. Yokoyama, J. I. Yoshikawa, P. van Loock, and A. Furusawa, Demonstration of a Controlled-Phase Gate for Continuous-Variable One-Way Quantum Computation, Phys. Rev. Lett. 107, 250501 (2011).

[8] X. Su, S. Hao, X. Deng, L. Ma, M. Wang, X. Jia, C. Xie, and K. Peng, Gate sequence for continuous variable one-way quantum computation, Nat. Commun. 4, 2828 (2013).

[9] S. Yokoyama, R. Ukai, S. C. Armstrong, C. Sornphiphatphong, T. Kaji, S. Suzuki, J. Yoshikawa, H. Yonezawa, N. C. Menicucci, and A. Furusawa, Ultra-large-scale continuousvariable cluster states multiplexed in the time domain, Nat. Photon. 7, 982 (2013).

[10] M. Chen, N. C. Menicucci, and O. Pfister, Experimental Realization of Multipartite Entanglement of 60 Modes of a Quantum Optical Frequency Comb, Phys. Rev. Lett. 112, 120505 (2014).

[11] J. I. Yoshikawa, S. Yokoyama, T. Kaji, C. Sornphiphatphong, Y. Shiozawa, K. Makino, and A. Furusawa, Invited article: Generation of one-million-mode continuous-variable cluster state by unlimited time-domain multiplexing, APL Photon. 1, 060801 (2016).

[12] R. N. Alexander, P. Wang, N. Sridhar, M. Chen, O. Pfister, and N. C. Menicucci, One-way quantum computing with arbitrarily large time-frequency continuous-variable cluster states from a single optical parametric oscillator, Phys. Rev. A 94, 032327 (2016).

[13] N. C. Menicucci, Temporal-mode continuous-variable cluster states using linear optics, Phys. Rev. A 83, 062314 (2011).

[14] N. C. Menicucci, S. T. Flammia, and O. Pfister, One-Way Quantum Computing in the Optical Frequency Comb, Phys. Rev. Lett. 101, 130501 (2008).

[15] S. T. Flammia, N. C. Menicucci, and O. Pfister, The optical frequency comb as a one-way quantum computer, J. Phys. B 42, 114009 (2009).

[16] R. N. Alexander, S. Yokoyama, A. Furusawa, and N. C. Menicucci, Universal quantum computation with temporalmode bilayer square lattices, Phys. Rev. A 97, 032302 (2018).

[17] M. V. Larsen, X. Guo, C. R. Breum, J. S. Neergaard-Nielsen, and U. L. Andersen, Deterministic generation of a twodimensional cluster state, Science 366, 369 (2019).

[18] W. Asavanant, Y. Shiozawa, S. Yokoyama, B. Charoensombutamon, H. Emura, R. N. Alexander, S. Takeda, J. Yoshikawa, N. C. Menicucci, H. Yonezawa, and A. Furusawa, Generation of time-domain-multiplexed two-dimensional cluster state, Science 366, 373 (2019).

[19] R. N. Alexander and N. C. Menicucci, Flexible quantum circuits using scalable continuous-variable cluster states, Phys. Rev. A 93, 062326 (2016).

[20] R. N. Alexander, N. C. Gabay, P. P. Rohde, and N. C. Menicucci, Measurement-Based Linear Optics, Phys. Rev. Lett. 118, 110503 (2017).

[21] R. N. Alexander, S. C. Armstrong, R. Ukai, and N. C. Menicucci, Noise analysis of single-mode Gaussian operations using continuous-variable cluster states, Phys. Rev. A 90, 062324 (2014).

[22] N. C. Menicucci, Fault-Tolerant Measurement-Based Quantum Computing with Continuous-Variable Cluster States, Phys. Rev. Lett. 112, 120504 (2014).
[23] P. T. Cochrane, G. J. Milburn, and W. J. Munro, Macroscopically distinct quantum-superposition states as a bosonic code for amplitude damping, Phys. Rev. A 59, 2631 (1999).

[24] A. P. Lund, T. C. Ralph, and H. L. Haselgrove, Fault-Tolerant Linear Optical Quantum Computing with Small-Amplitude Coherent States, Phys. Rev. Lett. 100, 030503 (2008).

[25] M. H. Michael, M. Silveri, R. T. Brierley, V. V. Albert, J. Salmilehto, L. Jiang, and S. M. Girvin, New Class of Quantum Error-Correcting Codes for a Bosonic Mode, Phys. Rev. X 6, 031006 (2016).

[26] V. V. Albert, K. Noh, K. Duivenvoorden, D. J. Young, R. T. Brierley, P. Reinhold, C. Vuillot, L. Li, C. Shen, S. M. Girvin, B. M. Terhal, and L. Jiang, Performance and structure of singlemode bosonic codes, Phys. Rev. A 97, 032346 (2018).

[27] D. Gottesman, A. Kitaev, and J. Preskill, Encoding a qubit in an oscillator, Phys. Rev. A 64, 012310 (2001).

[28] A. M. Steane, Overhead and noise threshold of fault-tolerant quantum error correction, Phys. Rev. A 68, 042322 (2003).

[29] E. Knill, Quantum computing with realistically noisy devices, Nature (London) 434, 39 (2005).

[30] A. Furusawa, J. L. Sørensen, S. L. Braunstein, C. A. Fuchs, H. J. Kimble, and E. S. Polzik, Unconditional quantum teleportation, Science 282, 706 (1998).

[31] N. C. Menicucci, S. T. Flammia, and P. van Loock, Graphical calculus for Gaussian pure states, Phys. Rev. A 83, 042335 (2011).

[32] R. Ukai, J. I. Yoshikawa, N. Iwata, P. van Loock, and A. Furusawa, Universal linear Bogoliubov transformations through one-way quantum computation, Phys. Rev. A 81, 032315 (2010).

[33] I. Tzitrin, J. E. Bourassa, N. C. Menicucci, and K. K. Sabapathy, Progress towards practical qubit computation using approximate Gottesman-Kitaev-Preskill codes, Phys. Rev. A 101, 032315 (2020).

[34] B. M. Terhal, J. Conrad, and C. Vuillot, Towards scalable bosonic quantum error correction, Quantum Sci. Technol. 5, 043001 (2020).

[35] S. Takeda, K. Takase, and A. Furusawa, On-demand photonic entanglement synthesizer, Sci. Adv. 5, eaaw4530 (2019).

[36] M. V. Larsen, X. Guo, C. R. Breum, J. S. Neergaard-Nielsen, and U. L. Andersen, Fiber coupled EPR-state generation using a single temporally multiplexed squeezed light source, npj Quantum Inf. 5, 46 (2019).

[37] H. Vahlbruch, M. Mehmet, K. Danzmann, and R. Schnabel, Detection of $15 \mathrm{~dB}$ Squeezed States of Light and their Application for the Absolute Calibration of Photoelectric Quantum Efficiency, Phys. Rev. Lett. 117, 110801 (2016).

[38] S. L. Braunstein, Squeezing as an irreducible resource, Phys. Rev. A 71, 055801 (2005).

[39] S. D. Bartlett, B. C. Sanders, S. L. Braunstein, and K. Nemoto, Efficient Classical Simulation of Continuous Variable Quantum Information Processes, Phys. Rev. Lett. 88, 097904 (2002).

[40] S. D. Bartlett and B. C. Sanders, Efficient Classical Simulation of Optical Quantum Information Circuits, Phys. Rev. Lett. 89, 207903 (2002).

[41] S. Lloyd and S. L. Braunstein, Quantum Computation over Continuous Variables, Phys. Rev. Lett. 82, 1784 (1999).

[42] U. L. Andersen, J. S. Neergaard-Nielsen, P. van Loock, and A. Furusawa, Hybrid discrete- and continuous-variable quantum information, Nat. Phys. 11, 713 (2015). 
[43] B. Hacker, S. Welte, S. Daiss, A. Shaukat, S. Ritter, L. Li, and G. Rempe, Deterministic creation of entangled atom-light Schrödinger-cat states, Nat. Photon. 13, 110 (2019).

[44] Y. Ra, A. Dufour, M. Walschaers, C. Jacquard, T. Michel, C. Fabre, and N. Treps, Non-Gaussian quantum states of a multimode light field, Nat. Phys. 16, 144 (2020).

[45] J. Hastrup, M. V. Larsen, J. S. Neergaard-Nielsen, N. C. Menicucci, and U. L. Andersen, Cubic phase gates are not suitable for non-Clifford operations on GKP states, arXiv:2009.05309.

[46] H. Yamasaki, T. Matsuura, and M. Koashi, Cost-reduced allGaussian universality with the Gottesman-Kitaev-Preskill code: Resource-theoretic approach to cost analysis, Phys. Rev. Res. 2, 023270 (2020).

[47] B. Q. Baragiola, G. Pantaleoni, R. N. Alexander, A. Karanjai, and N. C. Menicucci, All-Gaussian Universality and Fault Tolerance with the Gottesman-Kitaev-Preskill Code, Phys. Rev. Lett. 123, 200502 (2019).
[48] B. W. Walshe, L. J. Mensen, B. Q. Baragiola, and N. C. Menicucci, Robust fault tolerance for continuous-variable cluster states with excess antisqueezing, Phys. Rev. A 100, 010301(R) (2019).

[49] K. Fukui, A. Tomita, A. Okamoto, and K. Fujii, High-Threshold Fault-Tolerant Quantum Computation with Analog Quantum Error Correction, Phys. Rev. X 8, 021054 (2018).

[50] K. Noh and C. Chamberland, Fault-tolerant bosonic quantum error correction with the surface-Gottesman-Kitaev-Preskill code, Phys. Rev. A 101, 012316 (2020).

[51] B. H. Wu, R. N. Alexander, S. Liu, and Z. Zhang, Quantum computing with multidimensional continuous-variable cluster states in a scalable photonic platform, Phys. Rev. Res. 2, 023138 (2020).

[52] K. Fukui, W. Asavanant, and A. Furusawa, Temporal-mode continuous-variable three-dimensional cluster state for topologically protected measurement-based quantum computation, Phys. Rev. A 102, 032614 (2020). 